\title{
A KRYLOV SUBSPACE APPROACH TO PARAMETRIC INVERSION OF ELECTROMAGNETIC DATA BASED ON RESIDUAL MINIMIZATION
}

\author{
Master Thesis \\ to be defended publicly \\ on 21 June 2010 at 1:00 p.m. by
}

Edmond Balidemaj

Laboratory of Electromagnetic Research

Faculty of Electrical Engineering Mathematics and Computer Science

Delft University of Technology

Mekelweg 4, 2628 CD Delft The Netherlands

edmond@balidemaj.com

June 2010 
This graduation work was carried out at the Laboratory of Electromagnetic Research of the Delft University of Technology.

Committee members:

Prof. dr. T.D. Visser

Dr. Ir. R.F. Remis

Dr. Ir. M.D. Verweij

Dr. N.V. Budko

Dr. Ir. B.J. Kooij
Delft University of Technology, EEMCS, Chairman

Delft University of Technology, EEMCS, Supervisor

Delft University of Technology, EEMCS

Delft University of Technology, EEMCS

Delft University of Technology, EEMCS

Copyright (C)2010 by E. Balidemaj, Laboratory of Electromagnetic Research, Faculty of Electrical Engineering, Mathematics, and Computer Science, Delft University of Technology

All rights reserved. No part of this publication may be reproduced, stored in a retrieval system, or transmitted, in any form or by any means, electronic, mechanical, photocopying, recording or otherwise without prior written permission of the Laboratory of Electromagnetic Research. 


\section{Contents}

1 Introduction $\quad 1$

2 Statement of the Problem and Basic Equations 3

2.1 Statement of the Problem . . . . . . . . . . . . . 3

2.2 Maxwell's Equations for E-Polarized Fields . . . . . . . . . . 4

2.3 Integral Representations for the Scattered Field . . . . . . . 7

2.3 .1 Data Equation . . . . . . . . . . . 8

2.3 .2 Object Equation . . . . . . . . . . 8

2.4 Discretization of the Data and Object Equations . . . . . . . 9

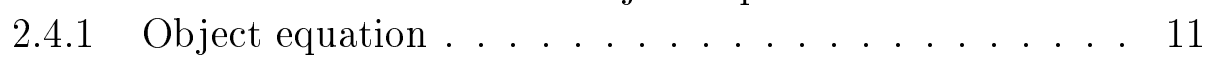

2.4 .2 Data equation . . . . . . . . . . . . 15

3 Numerical Methods Based on a Krylov Subspace 18

3.1 Introduction . . . . . . . . . . . . . . 18

3.2 Krylov Subspaces . . . . . . . . . . . . . . . . 18

3.3 Arnoldi Algorithm . . . . . . . . . . . . . . . 21

3.4 GMRES . . . . . . . . . . . . . . . . . 22

3.4.1 Restarted GMRES . . . . . . . . . . . 24

3.4 .2 Shifted GMRES . . . . . . . . . . 25

3.4.3 Restarted GMRES for Shifted Linear Systems . . . . 27

4 Numerical Results $\quad 31$

4.1 Introduction . . . . . . . . . . . . . . . 31

4.2 Configuration ....................... 31

4.3 Single Frequency Radiation Source . . . . . . . . . . . . 35

4.4 Multiple-Frequency Radiation Source . . . . . . . . . . 36

4.5 Effective Inversion . . . . . . . . . . . . . . 37

5 Conclusions \& Recommendations $\quad 41$ 
A Accepted Paper Progress in Electromagnetics Research Symposium (PIERS 2010), Cambridge, USA, on 5-8 July, 201043

B MATLAB Code $\quad 49$

$\begin{array}{ll}\text { References } & 59\end{array}$ 


\section{Chapter 1}

\section{Introduction}

Inverse scattering problems are usually solved using iterative inversion methods where at every inversion step forward problems are solved for many parameters. These methods may be computationally very expensive especially if little is known about the scatterer. If the scatterer is a homogeneous object then the forward problems can be solved efficiently by exploiting the shiftinvariance property of Krylov subspaces. To be more specific, the total field approximations corresponding to a whole range of contrast coefficients live in the same Krylov subspace. This requires a single run of the Krylov subspace method to construct all these fields. Previously[1] these field approximations were constructed using the Arnoldi algorithm in combination with a Full Orthogonalization (FO) approach. The drawback of this approach is that, for a given contrast, the norm of the residuals corresponding to successive field approximations is not necessarily a nonincreasing function.

In this report we present a method which ensures that the Euclidean norm of all residuals is minimum. Furthermore, it guarantees successive residuals of the field approximations, for a given contrast, to be a decreasing function. This is similar to the well known Generalized Minimum Residual or GMRES method. Using the presented method, we only need to solve small-scale least-squares problems for a whole range of contrast parameters. Storage problem becomes an issue if we are dealing with electrically large objects since all Krylov subspace vectors need to be stored. To remedy this, we introduce a restarted version of the above method as proposed in [2]. Restarting the method however, introduces another storage problem as the field approximations corresponding to each contrast coefficient need to be stored at every restart. To avoid this problem, we treat parts of the range of contrast coefficients at a time.

Using the approximated total electric field we compute the scattered field values in a straightforward manner. The true medium parameters are finally 
retrieved by inspecting an objective function which measures the discrepancy of the modeled scattered field value and the true scattered field value at the specified receiver location. The complete inversion method can be summarized as follows:

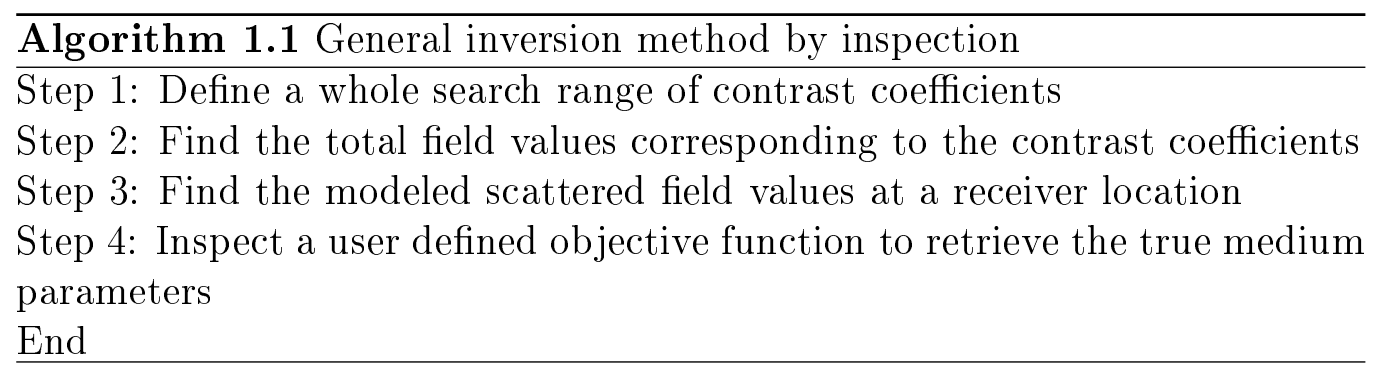

The presented method might as well be used to map the overall contrast function of an inhomogeneous scatterer to a homogeneous one. To this end, we try to match the scattered field due to a homogeneous object to the scattered field generated by an inhomogeneous object. This procedure is referred to as effective inversion and is important in many different areas most notably in effective medium theory.

This thesis is organized as follows. In Chapter 2 we derive the object and data equations and present their discretized equivalents. In Chapter 3 we present our iterative inversion algorithm and, finally, in Chapter 4 we illustrate the performance of the proposed method by a number of numerical examples. 


\section{Chapter 2}

\section{Statement of the Problem and Basic Equations}

\subsection{Statement of the Problem}

We consider E-polarized waves in a two-dimensional configuration that is invariant in the $z$-direction. A penetrable object occupies a finite domain $\mathbb{D}^{\text {sc }}$ and is characterized by a conductivity $\sigma^{\text {sc }}$, a permittivity $\varepsilon^{\text {sc }}$, and a permeability $\mu_{0}$. The object is located in vacuum and may be homogeneous ( $\sigma^{\mathrm{sc}}$ and $\varepsilon^{\mathrm{sc}}$ are constant) or inhomogeneous ( $\sigma^{\mathrm{sc}}$ and $\varepsilon^{\mathrm{sc}}$ are position dependent). The goal of this thesis is to obtain, in a nondestructive way, accurate constant (i.e. position-independent) estimates for the conductivity and permittivity of the object (see Figure 2.1). To this end, we illuminate the object by electromagnetic waves and measure the resulting scattered field. Having this field available, we now estimate the conductivity and permittivity of the object by minimizing a specific objective function. This function is a measure of the discrepancy between the true measured field and the scattered field at the receiver locations as generated by a homogeneous object with a constant conductivity and permittivity. Those constant values for which the objective function is minimum are taken as medium parameter estimates. Obviously, if the object is homogeneous then our problem amounts to solving a two-parameter inverse scattering problem. For an inhomogeneous object, however, we are essentially dealing with an effective inversion problem, since we are looking for constant medium parameters even if the object is inhomogeneous. 


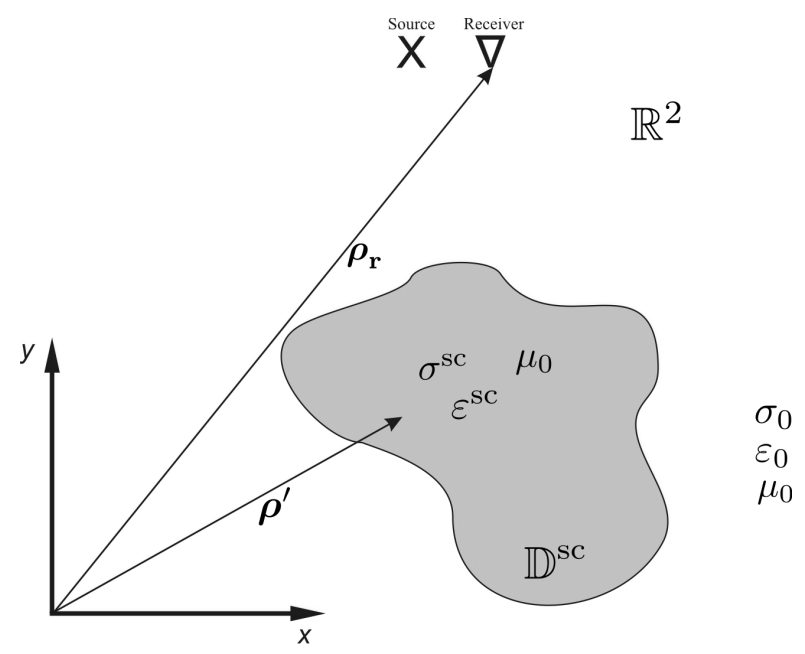

Figure 2.1: Two-dimensional configuration. The case where $\rho^{\prime} \in \mathbb{D}^{\text {sc }}$ and $\rho_{\mathbf{r}} \notin \mathbb{D}^{\text {sc }}$ is depicted.

\subsection{Maxwell's Equations for E-Polarized Fields}

We start with Maxwell's equations for E-polarized fields. In the frequencydomain, these equations are given by

$$
\begin{aligned}
\partial_{y} \hat{H}_{x}-\partial_{x} \hat{H}_{y}+\sigma \hat{E}_{z}-i \omega \varepsilon \hat{E}_{z} & =-\hat{J}_{z}^{\mathrm{ext}}, \\
\partial_{y} \hat{E}_{z}-i \omega \mu_{0} \hat{H}_{x} & =0, \\
-\partial_{x} \hat{E}_{z}-i \omega \mu_{0} \hat{H}_{y} & =0
\end{aligned}
$$

where $\hat{E}_{z}$ is the $z$-component of the electric field strength and $\hat{H}_{x}$ and $\hat{H}_{y}$ are the $x$ - and $y$-components of the magnetic field strength, see [3] and [4]. Furthermore, $\sigma$ is the conductivity of the medium in which the electromagnetic field is present, $\varepsilon$ is its permittivity, and $\mu_{0}$ its permeability. Finally, $\hat{J}_{z}^{\text {ext }}$ is the $z$-component of the external electric current density.

Since our configuration consists of an object embedded in vacuum, we setup a scattering formalism and exploit the linearity of Maxwell's equations. Specifically, we write the total electromagnetic field as a sum of an incident and a scattered field. The incident field is the field that is present if the object is absent and the scattered field takes the presence of the object into account. Starting with the incident field, its basic equations are 


$$
\begin{aligned}
\partial_{y} \hat{H}_{x}^{\mathrm{inc}}-\partial_{x} \hat{H}_{y}^{\mathrm{inc}}-i \omega \varepsilon_{0} \hat{E}_{z}^{\mathrm{inc}}=-\hat{J}_{z}^{\mathrm{ext}}, \\
\partial_{y} \hat{E}_{z}^{\mathrm{inc}}-i \omega \mu_{0} \hat{H}_{x}^{\mathrm{inc}}=0, \\
-\partial_{x} \hat{E}_{z}^{\mathrm{inc}}-i \omega \mu_{0} \hat{H}_{y}^{\mathrm{inc}}=0,
\end{aligned}
$$

if $\boldsymbol{\rho} \notin \mathbb{D}^{\text {sc }}$, and inside the object we have

$$
\begin{array}{r}
\partial_{y} \hat{H}_{x}^{\mathrm{inc}}-\partial_{x} \hat{H}_{y}^{\mathrm{inc}}-i \omega \varepsilon_{0} \hat{E}_{z}^{\mathrm{inc}}=0, \\
\partial_{y} \hat{E}_{z}^{\mathrm{inc}}-i \omega \mu_{0} \hat{H}_{x}^{\mathrm{inc}}=0, \\
-\partial_{x} \hat{E}_{z}^{\mathrm{inc}}-i \omega \mu_{0} \hat{H}_{y}^{\mathrm{inc}}=0,
\end{array}
$$

with $\rho \in \mathbb{D}^{\text {sc }}$. Similarly, for the total field outside the object domain $\mathbb{D}^{\text {sc }}$ we have

$$
\begin{aligned}
\partial_{y} \hat{H}_{x}-\partial_{x} \hat{H}_{y}-i \omega \varepsilon_{0} \hat{E}_{z} & =-\hat{J}_{z}^{\mathrm{ext}}, \\
\partial_{y} \hat{E}_{z}-i \omega \mu_{0} \hat{H}_{x} & =0, \\
-\partial_{x} \hat{E}_{z}-i \omega \mu_{0} \hat{H}_{y} & =0,
\end{aligned}
$$

with $\rho \notin \mathbb{D}^{\text {sc }}$, and for the total field inside the object domain

$$
\begin{array}{r}
\partial_{y} \hat{H}_{x}-\partial_{x} \hat{H}_{y}+\sigma^{\mathrm{sc}} \hat{E}_{z}-i \omega \varepsilon^{\mathrm{sc}} \hat{E}_{z}=0 \\
\partial_{y} \hat{E}_{z}-i \omega \mu_{0} \hat{H}_{x}=0 \\
-\partial_{x} \hat{E}_{z}-i \omega \mu_{0} \hat{H}_{y}=0
\end{array}
$$

with $\rho \in \mathbb{D}^{\text {sc }}$. As a first step towards the introduction of the scattered field, we rewrite Eq.(2.13) as

$$
\partial_{y} \hat{H}_{x}-\partial_{x} \hat{H}_{y}-i \omega \varepsilon_{0} \hat{E}_{z}=-\left[\sigma^{\mathrm{sc}}-i \omega\left(\varepsilon^{\mathrm{sc}}-\varepsilon_{0}\right)\right] \hat{E}_{z},
$$

which obviously holds for $\rho \in \mathbb{D}^{\text {sc }}$. For the total field inside the object, we now have

$$
\begin{aligned}
\partial_{y} \hat{H}_{x}-\partial_{x} \hat{H}_{y}-i \omega \varepsilon_{0} \hat{E}_{z} & =-\left[\sigma^{\mathrm{sc}}-i \omega\left(\varepsilon^{\mathrm{sc}}-\varepsilon_{0}\right)\right] \hat{E}_{z}, \\
\partial_{y} \hat{E}_{z}-i \omega \mu_{0} \hat{H}_{x} & =0 \\
-\partial_{x} \hat{E}_{z}-i \omega \mu_{0} \hat{H}_{y} & =0
\end{aligned}
$$


with $\rho \in \mathbb{D}^{\text {sc }}$. If we now subtract Eqs. (2.4)-(2.6) from Eqs. (2.10)-(2.12) and Eqs. (2.7)-(2.9) from Eqs. (2.16)-(2.18) we obtain

$$
\begin{array}{r}
\partial_{y} \hat{H}_{x}^{\mathrm{sc}}-\partial_{x} \hat{H}_{y}^{\mathrm{sc}}-i \omega \varepsilon_{0} \hat{E}_{z}^{\mathrm{sc}}=0, \\
\partial_{y} \hat{E}_{z}^{\mathrm{sc}}-i \omega \mu_{0} \hat{H}_{x}^{\mathrm{sc}}=0, \\
-\partial_{x} \hat{E}_{z}^{\mathrm{sc}}-i \omega \mu_{0} \hat{H}_{y}^{\mathrm{sc}}=0,
\end{array}
$$

if $\rho \notin \mathbb{D}^{\mathrm{sc}}$, and

$$
\begin{aligned}
\partial_{y} \hat{H}_{x}^{\mathrm{sc}}-\partial_{x} \hat{H}_{y}^{\mathrm{sc}}-i \omega \varepsilon_{0} \hat{E}_{z}^{\mathrm{sc}} & =-\left[\sigma^{\mathrm{sc}}-i \omega\left(\varepsilon^{\mathrm{sc}}-\varepsilon_{0}\right)\right] \hat{E}_{z}, \\
\partial_{y} \hat{E}_{z}^{\mathrm{sc}}-i \omega \mu_{0} \hat{H}_{x}^{\mathrm{sc}} & =0, \\
-\partial_{x} \hat{E}_{z}^{\mathrm{sc}}-i \omega \mu_{0} \hat{H}_{y}^{\mathrm{sc}} & =0,
\end{aligned}
$$

if $\boldsymbol{\rho} \in \mathbb{D}^{\mathrm{sc}}$, where we have introduced the scattered field as

$$
\begin{aligned}
\hat{H}_{x}^{\mathrm{sc}} & =\hat{H}_{x}-\hat{H}_{x}^{\mathrm{inc}}, \\
\hat{H}_{y}^{\mathrm{sc}} & =\hat{H}_{y}-\hat{H}_{y}^{\mathrm{inc}}, \\
\hat{E}_{z}^{\mathrm{sc}} & =\hat{E}_{z}-\hat{E}_{x}^{\mathrm{inc}} .
\end{aligned}
$$

Introducing the scattering source $\hat{J}_{z}^{\mathrm{sc}}$ as

$$
\hat{J}_{z}^{\mathrm{sc}}= \begin{cases}{\left[\sigma^{\mathrm{sc}}-i \omega\left(\varepsilon^{\mathrm{sc}}-\varepsilon_{0}\right)\right] \hat{E}_{z}} & \text { if } \boldsymbol{\rho} \in \mathbb{D}^{\mathrm{sc}}, \\ 0 & \text { if } \boldsymbol{\rho} \notin \mathbb{D}^{\mathrm{sc}},\end{cases}
$$

then Eqs. (2.19)-(2.24) can be written as

$$
\begin{aligned}
\partial_{y} \hat{H}_{x}^{\mathrm{sc}}-\partial_{x} \hat{H}_{y}^{\mathrm{sc}}-i \omega \varepsilon_{0} \hat{E}_{z}^{\mathrm{sc}}=-\hat{J}_{z}^{\mathrm{sc}}, \\
\partial_{y} \hat{E}_{z}^{\mathrm{sc}}-i \omega \mu_{0} \hat{H}_{x}^{\mathrm{sc}}=0 \\
-\partial_{x} \hat{E}_{z}^{\mathrm{sc}}-i \omega \mu_{0} \hat{H}_{y}^{\mathrm{sc}}=0
\end{aligned}
$$

with $\rho \in \mathbb{R}^{2}$. Finally, we note that the contrast source can also be written as

$$
\hat{J}_{z}^{\mathrm{sc}}= \begin{cases}-i \omega \varepsilon_{0} \chi(\boldsymbol{\rho}, \omega) \hat{E}_{z} & \text { if } \boldsymbol{\rho} \in \mathbb{D}^{\mathrm{sc}}, \\ 0 & \text { if } \boldsymbol{\rho} \notin \mathbb{D}^{\mathrm{sc}}\end{cases}
$$


where we have introduced the contrast function $\chi$ as

$$
\chi(\boldsymbol{\rho}, \omega)=\varepsilon_{\mathrm{r}}(\boldsymbol{\rho})-1+i \frac{\sigma^{\mathrm{sc}}(\boldsymbol{\rho})}{\omega \varepsilon_{0}},
$$

with relative permittivity $\varepsilon_{\mathrm{r}}(\boldsymbol{\rho})=\varepsilon^{\mathrm{sc}}(\boldsymbol{\rho}) / \varepsilon_{0}$.

\subsection{Integral Representations for the Scattered Field}

In this section, we derive an integral representation for the scattered electric field strength, starting from Eq. (2.29)-(2.31). First, we multiply Eq. (2.29) by $i \omega \varepsilon_{0}$. This gives

$$
\partial_{y}\left(i \omega \mu_{0} \hat{H}_{x}^{\mathrm{sc}}\right)-\partial_{x}\left(i \omega \mu_{0} \hat{H}_{y}^{\mathrm{sc}}\right)+k^{2} \hat{E}_{z}^{\mathrm{sc}}=-i \omega \mu_{0} \hat{J}_{z}^{\mathrm{sc}},
$$

where we have introduced the wave number $k$ as

$$
k=\omega / c_{0} \quad \text { with } c_{0}=\left(\varepsilon_{0} \mu_{0}\right)^{-1 / 2} .
$$

From Eqs. (2.30) and (2.31) it follows that

$$
i \omega \mu_{0} \hat{H}_{x}^{\mathrm{sc}}=\partial_{y} \hat{E}_{z}^{\mathrm{sc}} \quad \text { and } \quad i \omega \mu_{0} \hat{H}_{y}^{\mathrm{sc}}=-\partial_{x} \hat{E}_{z}^{\mathrm{sc}} .
$$

Substituting these equations in Eq. (2.34), we arrive at

$$
\left(\partial_{x}^{2}+\partial_{y}^{2}+k^{2}\right) \hat{E}_{z}^{\mathrm{sc}}=-i \omega \mu_{0} \hat{J}_{z}^{\mathrm{sc}} .
$$

To obtain $\hat{E}_{z}^{\mathrm{sc}}$, we need to solve Eq. (2.35). To this end, consider first the problem of finding the so-called Green's function $\hat{G}$ that satisfies

$$
\left(\partial_{x}^{2}+\partial_{y}^{2}+k^{2}\right) \hat{G}(\boldsymbol{\rho}, \omega)=-\delta(\boldsymbol{\rho}),
$$

and the condition that $\hat{G}$ should describe a wave that travels away from the origin $\boldsymbol{\rho}=\mathbf{0}$, where the Dirac delta function is active $(\hat{G}$ should satisfy the radiation condition). Using the superposition principle, it follows that $\hat{E}_{z}^{\mathrm{sc}}$ is given by

$$
\hat{E}_{z}^{\mathrm{sc}}(\boldsymbol{\rho}, \omega)=i \omega \mu_{0} \int_{\boldsymbol{\rho}^{\prime} \in \mathbb{D}^{\mathrm{sc}}} \hat{G}\left(\boldsymbol{\rho}-\boldsymbol{\rho}^{\prime}, \omega\right) \hat{J}_{z}^{\mathrm{sc}}\left(\boldsymbol{\rho}^{\prime}, \omega\right) d A,
$$

which holds for all $\boldsymbol{\rho} \in \mathbb{R}^{2}$. It can be shown that 


$$
\hat{G}(\boldsymbol{\rho}, \omega)=\frac{i}{4} H_{0}^{(1)}(k|\boldsymbol{\rho}|),
$$

where $H_{0}^{(1)}$ is the Hankel function of the first kind and of order zero. With this result we obtain

$$
\hat{E}_{z}^{\mathrm{sc}}(\boldsymbol{\rho}, \omega)=-\frac{\omega \mu_{0}}{4} \int_{\boldsymbol{\rho}^{\prime} \in \mathbb{D}^{\mathrm{sc}}} H_{0}^{(1)}\left(k\left|\boldsymbol{\rho}-\boldsymbol{\rho}^{\prime}\right|\right) \hat{J}_{z}^{\mathrm{sc}}\left(\boldsymbol{\rho}^{\prime}, \omega\right) d A,
$$

with $\boldsymbol{\rho} \in \mathbb{R}^{2}$. Finally, using Eq. (2.32) in the above equation we arrive at

$$
\begin{aligned}
\hat{E}_{z}^{\mathrm{sc}}(\boldsymbol{\rho}, \omega) & =-\frac{\omega \mu_{0}}{4} \cdot-i \omega \varepsilon_{0} \int_{\boldsymbol{\rho}^{\prime} \in \mathbb{D}^{\mathrm{sc}}} H_{0}^{(1)}\left(k\left|\boldsymbol{\rho}-\boldsymbol{\rho}^{\prime}\right|\right) \chi\left(\boldsymbol{\rho}^{\prime}, \omega\right) \hat{E}_{z}\left(\boldsymbol{\rho}^{\prime}, \omega\right) d A \\
& =\frac{i k^{2}}{4} \int_{\boldsymbol{\rho}^{\prime} \in \mathbb{D}^{\mathrm{sc}}} H_{0}^{(1)}\left(k\left|\boldsymbol{\rho}-\boldsymbol{\rho}^{\prime}\right|\right) \chi\left(\boldsymbol{\rho}^{\prime}, \omega\right) \hat{E}_{z}\left(\boldsymbol{\rho}^{\prime}, \omega\right) d A
\end{aligned}
$$

with $\rho \in \mathbb{R}^{2}$. This is the integral representation for the scattered electric field. By restricting the observation vector $\boldsymbol{\rho}$ to the position vectors of the receiver or the object domain, we obtain the data and object equation, respectively.

\subsubsection{Data Equation}

We use a single receiver to measure the electric field strength somewhere outside the object. Denoting the position vector of the receiver by $\boldsymbol{\rho}_{\mathbf{r}} \notin \mathbb{D}^{\text {sc }}$, we have for the scattered electric field strength at the receiver location, the integral representation

$$
\hat{E}_{z}^{\mathrm{sc}}\left(\boldsymbol{\rho}_{\mathbf{r}}, \omega\right)=\frac{i k^{2}}{4} \int_{\boldsymbol{\rho}^{\prime} \in \mathbb{D}^{\mathrm{sc}}} H_{0}^{(1)}\left(k\left|\boldsymbol{\rho}_{\mathbf{r}}-\boldsymbol{\rho}^{\prime}\right|\right) \chi\left(\boldsymbol{\rho}^{\prime}, \omega\right) \hat{E}_{z}\left(\boldsymbol{\rho}^{\prime}, \omega\right) d A .
$$

This equation is known as the data equation. It relates the scattered field at the receiver location to the total field inside the object. The latter field is unknown of course, but we do know that it must satisfy the so-called object equation.

\subsubsection{Object Equation}

The object equation is obtained from Eq. (2.27) by restricting the position vector to the object domain and using the definition of the scattered field. Specifically, we have 


$$
\hat{E}_{z}(\boldsymbol{\rho}, \omega)-\hat{E}_{z}^{\mathrm{sc}}(\boldsymbol{\rho}, \omega)=\hat{E}_{x}^{\mathrm{inc}}(\boldsymbol{\rho}, \omega),
$$

for $\rho \in \mathbb{D}^{\text {sc }}$ and substituting the integral representation for the scattered field, we obtain

$$
\hat{E}_{z}(\boldsymbol{\rho}, \omega)-\frac{i k^{2}}{4} \int_{\boldsymbol{\rho}^{\prime} \in \mathbb{D}^{\mathrm{sc}}} H_{0}^{(1)}\left(k\left|\boldsymbol{\rho}-\boldsymbol{\rho}^{\prime}\right|\right) \chi\left(\boldsymbol{\rho}^{\prime}, \omega\right) \hat{E}_{z}\left(\boldsymbol{\rho}^{\prime}, \omega\right) d A=\hat{E}_{z}^{\mathrm{inc}}(\boldsymbol{\rho}, \omega),
$$

with $\boldsymbol{\rho} \in \mathbb{D}^{\text {sc }}$. Equation (2.42) is known as the object equation. It is an integral equation of the second kind for the total electric field if the contrast function $\chi$ is known.

\subsection{Discretization of the Data and Object Equa- tions}

Since the data and object equations have to be evaluated on a computer we need to discretize equations (2.41) and (2.42) first. To make discretization as easy as possible we introduce a rectangular domain $\mathbb{S}^{\mathrm{d}}$ with sides $\ell_{x}$ and $\ell_{y}$, such that $\mathbb{D}^{\text {sc }} \subseteq \mathbb{S}^{\mathrm{d}}$. We then extend the domain of integration in Eqs. (2.41)-(2.42) to the introduced domain $\mathbb{S}^{\mathrm{d}}$. This is allowed since the contrast function $\chi(\boldsymbol{\rho}, \omega)=0$ for $\boldsymbol{\rho} \notin \mathbb{D}^{\mathrm{sc}}$. Hence the object equation for the introduced domain can be written as

$$
\hat{E}_{z}(\boldsymbol{\rho}, \omega)-\frac{i k^{2}}{4} \int_{\boldsymbol{\rho}^{\prime} \in \mathbb{S}^{\mathrm{d}}} H_{0}^{(1)}\left(k\left|\boldsymbol{\rho}-\boldsymbol{\rho}^{\prime}\right|\right) \chi\left(\boldsymbol{\rho}^{\prime}, \omega\right) \hat{E}_{z}\left(\boldsymbol{\rho}^{\prime}, \omega\right) d A=\hat{E}_{z}^{\mathrm{inc}}(\boldsymbol{\rho}, \omega),
$$

with $\rho \in \mathbb{S}^{\mathrm{d}}$ and the data equation as

$$
E_{z}^{\mathrm{sc}}\left(\boldsymbol{\rho}_{\mathbf{r}}, \omega\right)=\frac{i k^{2}}{4} \int_{\boldsymbol{\rho}^{\prime} \in \mathbb{S}^{d}} H_{0}^{(1)}\left(k\left|\boldsymbol{\rho}_{\mathbf{r}}-\boldsymbol{\rho}^{\prime}\right|\right) \chi\left(\boldsymbol{\rho}^{\prime}, \omega\right) E_{z}\left(\boldsymbol{\rho}^{\prime}, \omega\right) d A,
$$

with $\rho_{\mathbf{r}} \notin \mathbb{S}^{\mathrm{d}}$.

We introduce a uniform grid on the domain $\mathbb{S}^{\mathrm{d}}$ consisting of square cells with side lengths $\delta$ such that $N_{x} \delta=\ell_{x}$ and $N_{y} \delta=\ell_{y}$. The position vector of the center of a cell is given by 


$$
\boldsymbol{\rho}_{m n}=x_{m} \mathbf{i}_{m}+y_{n} \mathbf{i}_{n},
$$

for $m=1,2, \ldots, N_{x}$ and $n=1,2, \ldots, N_{y}$, where $x_{m}$ and $y_{n}$ are the coordinates of the center of a cell. Hence, we have

$$
x_{m}=\frac{\delta}{2}+(m-1) \delta \quad \text { and } \quad y_{n}=\frac{\delta}{2}+(n-1) \delta .
$$

The domain of a square cell is denoted by $\mathbb{S}_{m n}$ and we define it as

$$
\mathbb{S}_{m n}=\left\{(x, y) \in \mathbb{R}^{2} ; x_{m}-\frac{\delta}{2}<x<x_{m}+\frac{\delta}{2} \text { and } y_{n}-\frac{\delta}{2}<y<y_{n}+\frac{\delta}{2}\right\} .
$$

The described discretization procedure is depicted in Figure 2.2. Furthermore, we introduce the pulse functions

$$
\prod\left(x ; x_{m}\right)= \begin{cases}1 & \text { if } x_{m}-\frac{\delta}{2}<x<x_{m}+\frac{\delta}{2} \\ 0 & \text { otherwise }\end{cases}
$$

and

$$
\prod\left(y ; y_{n}\right)= \begin{cases}1 & \text { if } y_{n}-\frac{\delta}{2}<y<y_{n}+\frac{\delta}{2} \\ 0 & \text { otherwise }\end{cases}
$$

Clearly, we have

$$
\prod\left(x_{i} ; x_{m}\right)=\delta_{i, m} \quad \text { and } \quad \prod\left(y_{j} ; y_{n}\right)=\delta_{j, n}
$$

where we have used the Kronecker delta's defined as

$$
\delta_{i, m}=\left\{\begin{array}{ll}
1 & \text { if } i=m, \\
0 & \text { if } i \neq m,
\end{array} \quad \text { and } \quad \delta_{j, n}= \begin{cases}1 & \text { if } j=n, \\
0 & \text { if } j \neq n .\end{cases}\right.
$$

Using Eqs. (2.45)-(2.46) we approximate the total electric field strength by the pulse expansion

$$
e_{z}(\boldsymbol{\rho}, \omega)=\sum_{m=1}^{N_{x}} \sum_{n=1}^{N_{y}} \alpha_{m n}(\omega) \prod\left(x ; x_{m}\right) \prod\left(y ; y_{n}\right),
$$

and for a specific cell with position vector $\boldsymbol{\rho}=\boldsymbol{\rho}_{i j}$ we can write 


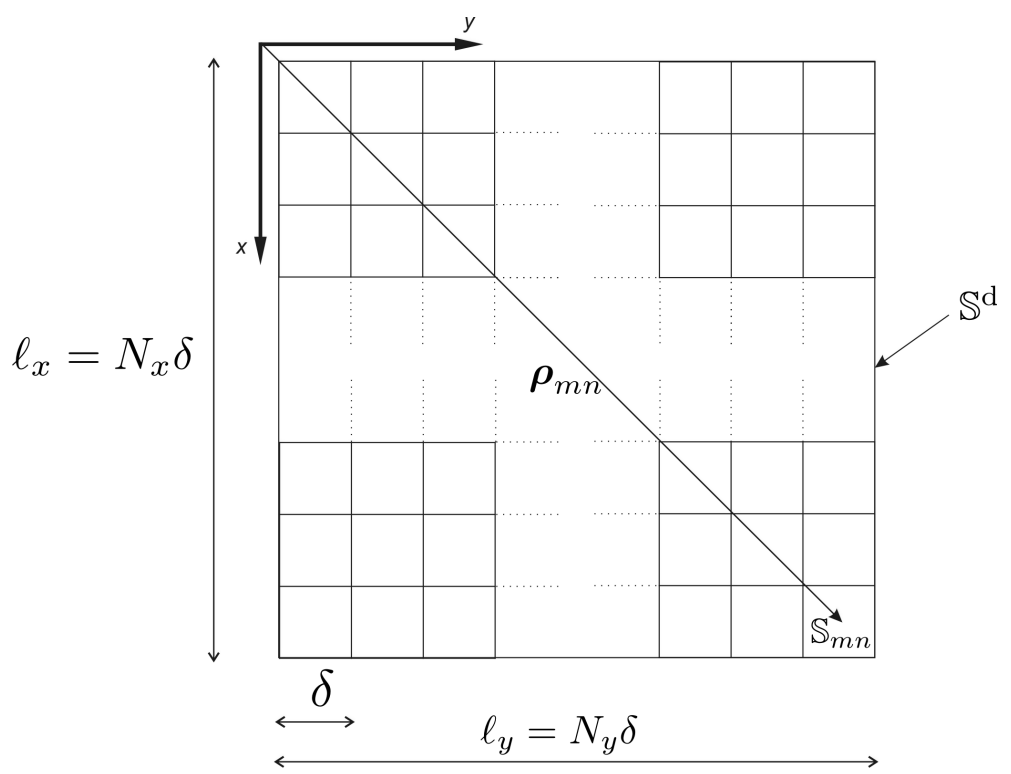

Figure 2.2: Domain $\mathbb{S}^{\mathrm{d}}$ and its discretization.

$$
\begin{aligned}
e_{z}\left(\boldsymbol{\rho}_{i j}, \omega\right) & =\sum_{m=1}^{N_{x}} \sum_{n=1}^{N_{y}} \alpha_{m n}(\omega) \prod\left(x_{i} ; x_{m}\right) \prod\left(y_{j} ; y_{n}\right) \\
& =\sum_{m=1}^{N_{x}} \sum_{n=1}^{N_{y}} \alpha_{m n}(\omega) \delta_{i, m} \delta_{j, n} \\
& =\alpha_{i j}(\omega)
\end{aligned}
$$

We observe that the electric field strength at the center of a specific square cell with position vector $\boldsymbol{\rho}_{i j}$ is equal to the expansion coefficient $\alpha_{i j}$.

\subsubsection{Object equation}

Now that we have defined the discrete electric field strength in Eq. (2.47) we continue discretizing the object equation (2.43). By substituting Eq. (2.47) in the object equation we have 


$$
\begin{aligned}
& \sum_{m=1}^{N_{x}} \sum_{n=1}^{N_{y}} \alpha_{m n}(\omega) \prod\left(x ; x_{m}\right) \prod\left(y ; y_{n}\right) \\
& -\frac{i k^{2}}{4} \int_{\boldsymbol{\rho}^{\prime} \in \mathbb{S}^{d}} H_{0}^{(1)}\left(k\left|\boldsymbol{\rho}-\boldsymbol{\rho}^{\prime}\right|\right) \chi\left(\boldsymbol{\rho}^{\prime}, \omega\right) \sum_{m=1}^{N_{x}} \sum_{n=1}^{N_{y}} \alpha_{m n}(\omega) \prod\left(x^{\prime} ; x_{m}\right) \prod\left(y^{\prime} ; y_{n}\right) d A \\
& =\hat{E}_{z}^{\mathrm{inc}}(\boldsymbol{\rho}, \omega) .
\end{aligned}
$$

We now interchange the order of integration and summation and take the expansion coefficient $\alpha_{m n}(\omega)$ out of the integral

$$
\begin{aligned}
& \sum_{m=1}^{N_{x}} \sum_{n=1}^{N_{y}} \alpha_{m n}(\omega) \prod\left(x ; x_{m}\right) \prod\left(y ; y_{n}\right) \\
& -\frac{i k^{2}}{4} \sum_{m=1}^{N_{x}} \sum_{n=1}^{N_{y}} \alpha_{m n}(\omega) \int_{\boldsymbol{\rho}^{\prime} \in \mathbb{S}^{d}} H_{0}^{(1)}\left(k\left|\boldsymbol{\rho}-\boldsymbol{\rho}^{\prime}\right|\right) \chi\left(\boldsymbol{\rho}^{\prime}, \omega\right) \prod\left(x^{\prime} ; x_{m}\right) \prod\left(y^{\prime} ; y_{n}\right) d A \\
& =\hat{E}_{z}^{\mathrm{inc}}(\boldsymbol{\rho}, \omega)
\end{aligned}
$$

with $\boldsymbol{\rho} \in \mathbb{S}^{\mathrm{d}}$. Since the summation signs are in front of the integral we do not need to integrate over the whole domain $\mathbb{S}^{\mathrm{d}}$ at every summation step as the the pulse functions, $\prod\left(x^{\prime} ; x_{m}\right)$ and $\prod\left(y^{\prime} ; y_{n}\right)$, are both zero if $\boldsymbol{\rho}^{\prime} \notin \mathbb{S}_{m n}$. So we might as well integrate over the smaller domain $\mathbb{S}_{m n}$ at every summation step. The above expression simplifies to

$$
\begin{aligned}
& \sum_{m=1}^{N_{x}} \sum_{n=1}^{N_{y}} \alpha_{m n}(\omega) \prod\left(x ; x_{m}\right) \prod\left(y ; y_{n}\right) \\
& -\frac{i k^{2}}{4} \sum_{m=1}^{N_{x}} \sum_{n=1}^{N_{y}} \alpha_{m n}(\omega) \int_{\boldsymbol{\rho}^{\prime} \in \mathbb{S}_{m n}} H_{0}^{(1)}\left(k\left|\boldsymbol{\rho}-\boldsymbol{\rho}^{\prime}\right|\right) \chi\left(\boldsymbol{\rho}^{\prime}, \omega\right) d A=\hat{E}_{z}^{\mathrm{inc}}(\boldsymbol{\rho}, \omega) .
\end{aligned}
$$

Let us now write the above equation for a specific position within domain $\mathbb{S}^{\mathrm{d}}$, namely, a point with position vector $\boldsymbol{\rho}=\boldsymbol{\rho}_{i j}$. We then have for the object equation 
$\alpha_{i j}(\omega)-\frac{i k^{2}}{4} \sum_{m=1}^{N_{x}} \sum_{n=1}^{N_{y}} \alpha_{m n}(\omega) \int_{\boldsymbol{\rho}^{\prime} \in \mathbb{S}_{m n}} H_{0}^{(1)}\left(k\left|\boldsymbol{\rho}_{i j}-\boldsymbol{\rho}^{\prime}\right|\right) \chi\left(\boldsymbol{\rho}^{\prime}, \omega\right) d A=\hat{E}_{z}^{\mathrm{inc}}\left(\boldsymbol{\rho}_{i j}, \omega\right)$,

where we have used Eq. (2.49) for the first term on the left-hand side of Eq. (2.50). Furthermore, the contrast function is assumed to be cell-wise constant, thus, the contrast function over the whole square cell of size $\delta$ is constant. Hence, we write

$$
\chi(\boldsymbol{\rho}, \omega) \approx \chi_{m n}(\omega) \quad \text { for } \boldsymbol{\rho} \in \mathbb{S}_{m n},
$$

where $\chi_{m n}(\omega)$ is the contrast function value for cell $\mathbb{S}_{m n}$. Finally, we set

$$
G_{m n i j}(\omega)=\frac{i k^{2}}{4} \int_{\boldsymbol{\rho}^{\prime} \in \mathbb{S}_{m n}} H_{0}^{(1)}\left(k\left|\boldsymbol{\rho}_{i j}-\boldsymbol{\rho}^{\prime}\right|\right) d A,
$$

and arrive at the following expression for the object equation

$$
\alpha_{i j}(\omega)-\frac{i k^{2}}{4} \sum_{m=1}^{N_{x}} \sum_{n=1}^{N_{y}} \alpha_{m n}(\omega) \chi_{m n}(\omega) G_{m n i j}(\omega)=\hat{E}_{z}^{\mathrm{inc}}\left(\boldsymbol{\rho}_{i j}, \omega\right) .
$$

We go back to Eq. (2.53) and be more specific in how we approximate the integral. First, we notice that the integrand behaves nicely for the case where $\boldsymbol{\rho}_{i j}$ is the position vector of the center of cell $\mathbb{S}_{i j}$, since $\boldsymbol{\rho}^{\prime}$ is running over a different cell, namely $\mathbb{S}_{m n}$. Consequently, we can approximate the integral by a simple midpoint rule. We obtain

$$
\begin{aligned}
G_{m n i j}(\omega) & \approx \frac{i k^{2}}{4} \delta^{2} H_{0}^{(1)}\left(k\left|\boldsymbol{\rho}_{i j}-\boldsymbol{\rho}_{m n}\right|\right) \\
& =\frac{i(k \delta)^{2}}{4} H_{0}^{(1)}\left[k \sqrt{\left(x_{i}-x_{m}\right)^{2}-\left(y_{j}-y_{n}\right)^{2}}\right] \\
& =\frac{i(k \delta)^{2}}{4} H_{0}^{(1)}\left[k \delta \sqrt{(i-m)^{2}-(j-n)^{2}}\right] .
\end{aligned}
$$

These elements of $G_{m n i j}(\omega)$, where $i \neq m$ and $j \neq n$, are called the nonself elements. For the self elements on the other hand, the case when $i=m$ and $j=n$, the approach is not as straightforward since then the integrand of Eq. (2.53) has a singularity. Specifically, we have 


$$
-i H_{0}^{(1)}(z) \sim \frac{2}{\pi} \ln (z) \quad \text { as } z \rightarrow 0,
$$

see [5]. The integrand is integrable, however. Our approach is to approximate the integral over a circular disc with radius $a$ having the same area as the cell $\mathbb{S}_{m n}$. The area of the circular disc is $\pi a^{2}$ which should be equal to the area of the square cell $\mathbb{S}_{m n}$ with size $\delta$. Thus $\delta^{2}=\pi a^{2}$ for radius $a=\frac{\delta}{\sqrt{\pi}}$. The center of the circular disk we take to be the same as that of the square disk being $\boldsymbol{\rho}_{m n}$ and the circular domain we denote by $\mathbb{D}_{m n}$. We now have

$$
\begin{aligned}
\int_{\boldsymbol{\rho}^{\prime} \in \mathbb{S}_{m n}} H_{0}^{(1)}\left(k\left|\boldsymbol{\rho}_{m n}-\boldsymbol{\rho}^{\prime}\right|\right) d A & \approx \int_{\boldsymbol{\rho}^{\prime} \in \mathbb{D}_{m n}} H_{0}^{(1)}\left(k\left|\boldsymbol{\rho}_{m n}-\boldsymbol{\rho}^{\prime}\right|\right) d A \\
& =\int_{\varphi=0}^{2 \pi} \int_{r=0}^{a} H_{0}^{(1)}(k r) r d r d \varphi \\
& =2 \pi \int_{r=0}^{a} H_{0}^{(1)}(k r) r d r \\
& =2 \pi k^{-2} \int_{z=0}^{k a} z H_{0}^{(1)}(z) d z \\
& =2 \pi k^{-2} \int_{z=0}^{k a} \frac{d}{d z}\left[z H_{1}^{(1)}(z)\right] d z \\
& =2 \pi k^{-2}\left[k a H_{1}^{(1)}(k a)+i \frac{2}{\pi}\right],
\end{aligned}
$$

where we have used the the expression

$$
z H_{0}^{(1)}(z)=\frac{d}{d z}\left[z H_{1}^{(1)}(z)\right]
$$

see [5]. Substituting Eq. (2.55) in Eq. (2.53), we have

$$
\begin{aligned}
G_{m n i j}(\omega) & \approx \frac{i k^{2}}{4} \cdot 2 \pi k^{-2}\left[k a H_{1}^{(1)}(k a)+i \frac{2}{\pi}\right] \\
& =\frac{i \pi}{2} k a H_{1}^{(1)}(k a)-1,
\end{aligned}
$$

for $i=m$ and $j=n$. These elements are usually referred to as self-elements. 
Let us now give the object equation in a matrix-vector notation. We start by arranging all unknown field values in an $N_{x} N_{y}-$ by -1 vector

$\mathbf{u}=\operatorname{vec}\left(\alpha_{i j}\right)=\left[\alpha_{11}, \alpha_{21}, \ldots, \alpha_{N_{x} 1}, \alpha_{12}, \alpha_{21}, \ldots, \alpha_{N_{x} 2}, \ldots, \alpha_{1 N_{y}}, \alpha_{2 N_{y}}, \ldots, \alpha_{N_{x} N_{y}}\right]^{\top}$

Similarly, the known incident field values are stored in an $N_{x} N_{y}-$ by -1 vector

$$
\mathbf{u}^{\mathrm{inc}}=\operatorname{vec}\left[\hat{E}_{z}^{\mathrm{inc}}\left(\boldsymbol{\rho}_{i j}, \omega\right)\right] .
$$

The object equation can now be written in matrix form as

$$
[\mathbf{I}-\mathbf{G X}] \mathbf{u}=\mathbf{u}^{\mathrm{inc}}
$$

with I the identity matrix of order $N_{x} N_{y}$ and $\mathbf{G}$ a complex square matrix of order $N_{x} N_{y}$, whose elements are given by

$$
G_{m n i j}=g_{i+(j-1) N_{x}, m+(n-1) N_{x}},
$$

for $i, m=1,2, \ldots, N_{x}$ and $j, n=1,2, \ldots, N_{y}$. Notice that the action of matrix $\mathbf{G}$ on a vector can be computed at "FFT speed", since matrix $\mathbf{G}$ has a block Toeplitz structure. Finally, matrix $\mathbf{X}$ is a diagonal matrix with the complex contrast function values on its diagonal. Specifically, we have

$$
\mathbf{X}=\operatorname{diag}(\mathbf{c}) \quad \text { with } \mathbf{c}=\operatorname{vec}\left(\chi_{m n}(\omega)\right) .
$$

Equation (2.57) is the discretized object equation in its final form and serves as one of the building blocks of our effective inversion scheme.

\subsubsection{Data equation}

The same approach is followed to discretize the data equation (2.44) repeated here

$$
\hat{E}_{z}^{\mathrm{sc}}\left(\boldsymbol{\rho}_{\mathbf{r}}, \omega\right)=\frac{i k^{2}}{4} \int_{\boldsymbol{\rho}^{\prime} \in \mathbb{S}^{d}} H_{0}^{(1)}\left(k\left|\boldsymbol{\rho}_{\mathbf{r}}-\boldsymbol{\rho}^{\prime}\right|\right) \chi\left(\boldsymbol{\rho}^{\prime}, \omega\right) E_{z}\left(\boldsymbol{\rho}^{\prime}, \omega\right) d A,
$$

with $\rho_{\mathbf{r}} \notin \mathbb{S}^{d}$. We substitute the pulse expansion for the electric field strength in above equation and obtain 


$$
\begin{aligned}
& \hat{E}_{z}^{\mathrm{sc}}\left(\boldsymbol{\rho}_{\mathbf{r}}, \omega\right) \approx \\
& \frac{i k^{2}}{4} \int_{\boldsymbol{\rho}^{\prime} \in \mathbb{S}^{d}} H_{0}^{(1)}\left(k\left|\boldsymbol{\rho}_{r}-\boldsymbol{\rho}^{\prime}\right|\right) \chi\left(\boldsymbol{\rho}^{\prime}, \omega\right) \sum_{m=1}^{N_{x}} \sum_{n=1}^{N_{y}} \alpha_{m n}(\omega) \prod\left(x^{\prime} ; x_{m}\right) \prod\left(y^{\prime} ; y_{n}\right) d A \\
& =\frac{i k^{2}}{4} \sum_{m=1}^{N_{x}} \sum_{n=1}^{N_{y}} \alpha_{m n}(\omega) \int_{\boldsymbol{\rho}^{\prime} \in \mathbb{S}^{d}} H_{0}^{(1)}\left(k\left|\boldsymbol{\rho}_{r}-\boldsymbol{\rho}^{\prime}\right|\right) \chi\left(\boldsymbol{\rho}^{\prime}, \omega\right) \prod\left(x^{\prime} ; x_{m}\right) \prod\left(y^{\prime} ; y_{n}\right) d A,
\end{aligned}
$$

where we again have interchanged the order of summation and integration. Assuming a cell-wise constant contrast function, the above expression simplifies to

$$
\hat{E}_{z}^{\mathrm{sc}}\left(\boldsymbol{\rho}_{\mathbf{r}}, \omega\right) \approx \frac{i k^{2}}{4} \sum_{m=1}^{N_{x}} \sum_{n=1}^{N_{y}} \alpha_{m n}(\omega) \chi_{m n}(\omega) \int_{\boldsymbol{\rho}^{\prime} \in \mathbb{S}_{m n}} H_{0}^{(1)}\left(k\left|\boldsymbol{\rho}_{r}-\boldsymbol{\rho}^{\prime}\right|\right) d A .
$$

Approximating the integral by the simple midpoint rule, we obtain

$$
\hat{E}_{z}^{\mathrm{sc}}\left(\boldsymbol{\rho}_{\mathbf{r}}, \omega\right) \approx \frac{i(k \delta)^{2}}{4} \sum_{m=1}^{N_{x}} \sum_{n=1}^{N_{y}} \alpha_{m n}(\omega) \chi_{m n}(\omega) H_{0}^{(1)}\left(k\left|\boldsymbol{\rho}_{r}-\boldsymbol{\rho}_{m n}\right|\right) .
$$

We can now give a matrix-vector representation of Eq. (2.59). In particular, we have

$$
\hat{E}_{z}^{\mathrm{sc}}\left(\boldsymbol{\rho}_{\mathbf{r}}, \omega\right) \approx \frac{i(k \delta)^{2}}{4} \mathbf{r}^{\top} \mathbf{X u},
$$

where we have introduced the receiver vector

$$
\mathbf{r}=\operatorname{vec}\left[H_{0}^{(1)}\left(k\left|\boldsymbol{\rho}_{r}-\boldsymbol{\rho}_{m n}\right|\right)\right] .
$$

Furthermore, we used $\mathbf{u}$ for the total electric field strength and matrix $\mathbf{X}$ for the contrast function as defined in the last subsection in Eqs. (2.56) and (2.58), respectively.

Let us now return to the inverse scattering problem and describe it with the use of the discretized object and data equations as defined in Eqs. (2.57) and (2.60), respectively. Recall that the task is to find matrix $\mathbf{X}$ where only $\mathbf{u}^{\text {inc }}$ and $\hat{E}_{z}^{\mathrm{sc}}$ are known. If we write Eq. (2.57) as 


$$
\mathbf{u}=[\mathbf{I}-\mathbf{G X}]^{-1} \mathbf{u}^{\text {inc }},
$$

and substitute it in Eq. (2.60), we get

$$
\hat{E}_{z}^{\mathrm{sc}}\left(\boldsymbol{\rho}_{\mathbf{r}}, \omega\right) \approx \frac{i(k \delta)^{2}}{4} \mathbf{r}^{\boldsymbol{\top}} \mathbf{X}[\mathbf{I}-\mathbf{G} \mathbf{X}]^{-1} \mathbf{u}^{\mathrm{inc}}=: u^{\mathrm{sc}},
$$

and we refer to $u^{\mathrm{sc}}$ as modeled scattered field data. It is obvious that the inverse scattering problem is nonlinear in $\mathbf{X}$ as the inverse matrix on the right-hand side depends on $\mathbf{X}$. In the next chapter, we describe how we solve this inverse problem for the particular case where the contrast is constant. 


\section{Chapter 3}

\section{Numerical Methods Based on a Krylov Subspace}

\subsection{Introduction}

The large computational power needed to solve large linear systems has made the use of iterative methods inevitable. Consider the linear system

$$
\mathrm{Ax}=\mathbf{b}
$$

with $\mathbf{A} \in \mathbb{C}^{n \times n}$ a non-singular complex matrix and $\mathbf{x}, \mathbf{b} \in \mathbb{C}^{n}$. If matrix $\mathbf{A}$ is large, finding the exact solution $\mathbf{x}=\mathbf{A}^{-1} \mathbf{b}$ is not possible with direct methods. Iterative methods instead seek an approximation $\mathbf{x}_{m} \approx \mathbf{x}$ in $m$ steps where $m \ll n$.

A well known iterative method for solving large linear system is GMRES and in this chapter we focus on this method. GMRES is based on Krylov subspaces and in Section 3.3 we describe how this subspace is generated by the use of the Arnoldi algorithm. Thereafter, we present the GMRES algorithm and a few variations of it, ending with the Restarted ShiftedGMRES algorithm.

\subsection{Krylov Subspaces}

Recall that in a general inverse scattering problem, our task is to find the contrast function $\mathbf{X}$ that satisfies equation (2.61). Here, we simplify this general inverse problem by considering objects with a homogeneous contrast only. Matrix $\mathbf{X}$ then simplifies to $\mathbf{X}=\chi \mathbf{I}$, where $\chi$ is a a complex scalar. The expression for the modeled scattered field now simplifies to 


$$
u^{\mathrm{sc}}=\frac{i(k \delta)^{2}}{4} \mathbf{r}^{\top} \chi \mathbf{u}
$$

where

$$
\mathbf{u}=[\mathbf{I}-\chi \mathbf{G}]^{-1} \mathbf{u}^{\text {inc }} .
$$

The way we retrieve the contrast function is by finding the total field values $\mathbf{u}_{1}, \mathbf{u}_{2}, \ldots, \mathbf{u}_{k}$ corresponding to a set of contrast functions $\chi_{1}, \chi_{2}, \ldots, \chi_{k}$, respectively. This we do by solving the object equation (3.3), rewritten as,

$$
\left[\mathbf{I}-\chi_{i} \mathbf{G}\right] \mathbf{u}_{i}=\mathbf{u}^{\text {inc }} \quad \text { for } i=1,2, \ldots, k,
$$

using the GMRES method explained later on in this chapter. Having obtained $\mathbf{u}_{1}, \mathbf{u}_{2}, \ldots, \mathbf{u}_{k}$ we substitute each of these field values in Eq. (3.2) to obtain the corresponding modeled scattered fields $u_{1}^{\mathrm{sc}}, u_{2}^{\mathrm{sc}}, \ldots, u_{k}^{\mathrm{sc}}$ at the receiver location $\boldsymbol{\rho}=\boldsymbol{\rho}_{\mathrm{r}}$. All these modeled scattered fields are compared with the true measured field $E_{z}^{\mathrm{sc}}\left(\boldsymbol{\rho}_{\mathbf{r}}, \omega\right)$ using the following objective function

$$
F_{i}=\frac{\left|\hat{E}_{z}^{\mathrm{sc}}\left(\boldsymbol{\rho}_{\mathbf{r}}, \omega\right)-u_{i}^{\mathrm{sc}}(\omega)\right|^{2}}{\left|\hat{E}_{z}^{\mathrm{sc}}\left(\boldsymbol{\rho}_{\mathbf{r}}, \omega\right)\right|^{2}},
$$

with $i=1,2, \ldots, k$. The modeled scattered field $u_{i}^{\text {sc }}$ for which $F_{i}<\epsilon$, where $\epsilon>0$ is a user specified tolerance, gives us the solution to our inverse problem. Since the total field vectors $\mathbf{u}_{i}$ are of size $n$, it is impractical to save them. Therefore, the described procedure should be implemented in such a way that the steps are carried out for one contrast function at a time. Solving the inverse scattering problem as described above we have the following algorithm:

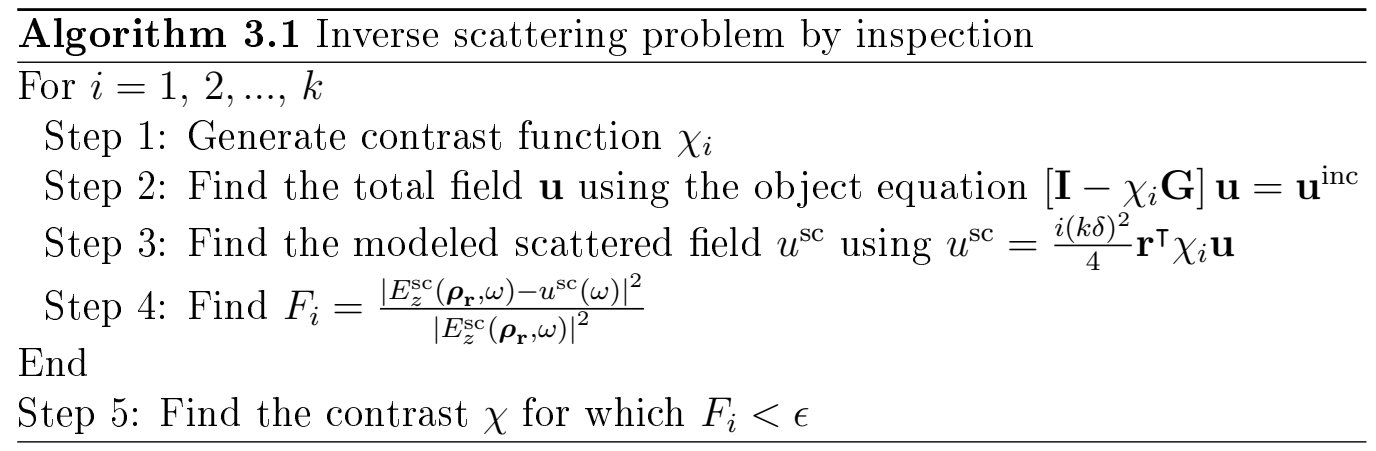


The most expensive computations are carried out in Step 2 as there is to be dealt with the large square matrix $\left[\mathbf{I}-\chi_{i} \mathbf{G}\right]$ of size $n$. Solving the object equation is therefore not done directly, as it requires $O\left(n^{3}\right)$ work, instead, iterative methods such as Krylov subspace methods are used yielding an approximate solution of the total field $\mathbf{u}$. These Krylov subspace methods approximate the exact solution $\mathbf{u}=[\mathbf{I}-\chi \mathbf{G}]^{-1} \mathbf{u}^{\text {inc }}$ by $\mathbf{u}_{m}=p_{m-1}([\mathbf{I}-\chi \mathbf{G}]) \mathbf{u}^{\text {inc }}$ where $p_{m-1}$ is a polynomial of degree $\leq m-1$. If we denote $[\mathbf{I}-\chi \mathbf{G}]$ by matrix $\mathbf{A}, \mathbf{u}^{\text {inc }}$ by vector $\mathbf{b}, \mathbf{u}$ by $\mathbf{x}$ and $\mathbf{u}_{m}$ by $\mathbf{x}_{m}$ we have

$$
\mathbf{x}=\mathbf{A}^{-1} \mathbf{b} \approx p_{m-1}(\mathbf{A}) \mathbf{b}=\mathbf{x}_{m} .
$$

It is obvious that vector $\mathbf{x}_{m}$ can be written as a linear combination of the vectors $\mathbf{b}, \mathbf{A} \mathbf{b}, \mathbf{A}^{2} \mathbf{b}, \ldots, \mathbf{A}^{m-1} \mathbf{b}$. Generating each of these vectors requires a matrix-vector product of $\mathbf{A}$ with the previous vector. Recall that in our case these matrix-vector products can be done at "FFT speed" as we stated earlier in the previous chapter. The linear subspace spanned by the $m$ vectors is called the order- $m$ Krylov subspace and since $\mathbf{x}_{m}$ lives in this subspace we have

$$
\mathbf{x}_{m} \in \mathcal{K}_{m}(\mathbf{A}, \mathbf{b})=\operatorname{span}\left\{\mathbf{b}, \mathbf{A} \mathbf{b}, \mathbf{A}^{2} \mathbf{b}, \ldots, \mathbf{A}^{m-1} \mathbf{b}\right\} .
$$

As these vectors tend to become linearly dependent some orthogonalization scheme must be used. A well known scheme for general matrices is Arnoldi which iteratively generates the basis vectors of the Krylov subspace as we see in the next section.

If there is a priori knowledge of the solution we can then exploit that by seeking for the solution $\mathbf{x}_{m}$ in the affine space $\mathbf{x}_{0}+\mathcal{K}_{m}\left(\mathbf{A}, \mathbf{r}_{0}\right)$. Thus

$$
\mathbf{x}_{m} \in \mathbf{x}_{0}+\mathcal{K}_{m}\left(\mathbf{A}, \mathbf{r}_{0}\right) \quad \text { with } \mathbf{r}_{0}=\mathbf{b}-\mathbf{A} \mathbf{x}_{0},
$$

where $\mathbf{x}_{0}$ is the initial guess and $\mathbf{r}_{0}$ the initial residual. We include the case $\mathbf{x}_{0} \neq \mathbf{0}$, since this emerges when considering restarts as we see in Subsection 3.4.1. Notice that for $\mathbf{x}_{0}=\mathbf{0}$ we have $\mathbf{r}_{0}=\mathbf{b}$ and thus Eq. (3.6) holds in general.

Since we are considering homogeneous objects with contrast function $\chi \in \mathbb{C}$, the square matrix $[\mathbf{I}-\chi \mathbf{G}]$ can be seen as a shifted version of the matrix G. As we see in Subsection 3.4.2, the approximate solutions $\mathbf{x}_{m}^{i}$ corresponding to contrast functions $\chi_{i}$, for $i=1,2, \ldots, k$, live in the very same Krylov subspace if we take the initial guess $\mathbf{x}_{0}^{i}=\mathbf{0}$. Hence

$$
\mathbf{x}_{m}^{i} \in \mathbf{x}_{0}^{i}+\mathcal{K}_{m}\left(\mathbf{A}, \mathbf{r}_{0}\right) \quad \text { if } \mathbf{x}_{0}^{i}=\mathbf{0} \text { for } i=1,2, \ldots, k .
$$

Making use of this shift-invariance property of Krylov subspaces helps us to drastically decrease the computational cost. Furthermore, in Subsection 
3.4.3 we extend this approach and present a method where it is possible to use non-zero $\mathbf{x}_{0}^{i}$ and still exploit the shift-invariance property of the Krylov subspaces.

In what follows, we present our results in their most general form by considering the linear system (3.1) with $\mathbf{A} \in \mathbb{C}^{n \times n}$ being a general non-singular complex matrix and $\mathbf{x}, \mathbf{b} \in \mathbb{C}^{n}$. Subsequently, we link the object equation (3.3) to the general linear system equation (3.1) so that implementation of the presented algorithms is straightforward.

\subsection{Arnoldi Algorithm}

The algorithm was first introduced by Arnoldi in 1951 to reduce a dense matrix into Hessenberg form so that approximations of some of its eigenvalues could be obtained. It was shown later that the approximated eigenvalues are the ones that are usually near the edge of the spectrum of the original matrix. In practice, these eigenvalues are precisely the ones that are of main interest in most applications. The Arnoldi algorithm is also very suitable to create an orthonormal basis of the Krylov subspace $\mathcal{K}_{m}$ in $m$-iteration steps, a property on which we focus in this section.

The modified Gram-Schmidt version of the Arnoldi algorithm, see [6], is as follows:

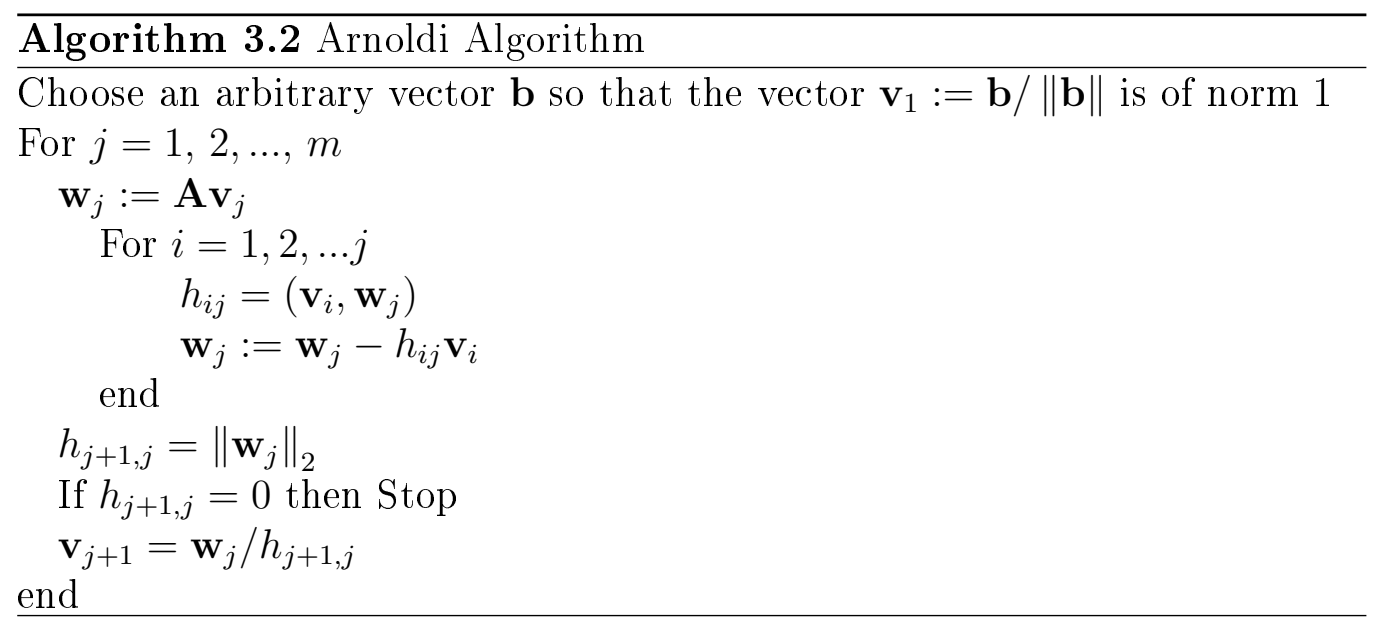

If we look at the $m$-th iteration of the algorithm, we can write the last vector $\mathbf{w}_{m}=\mathbf{A} \mathbf{v}_{m}$ as

$$
\mathbf{A} \mathbf{v}_{m}=h_{1 m} \mathbf{v}_{1}+\cdots+h_{m m} \mathbf{v}_{m}+h_{m+1, m} \mathbf{v}_{m+1},
$$

which can be written in matrix form as 


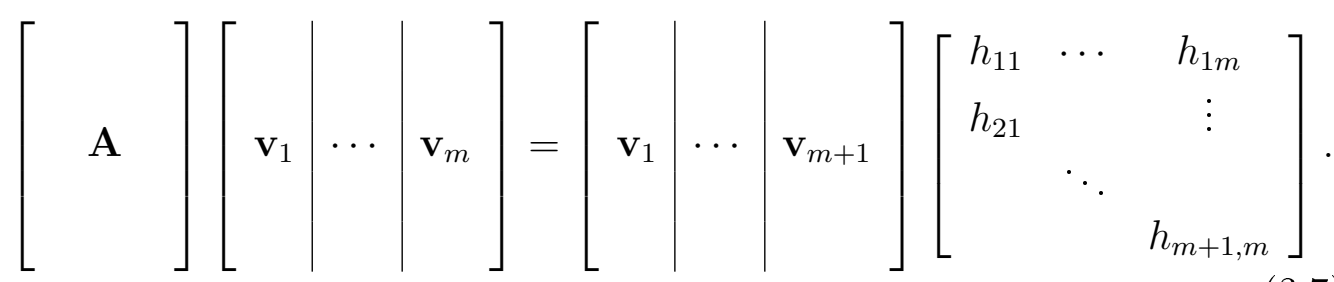

Let us now denote by $\mathbf{V}_{m}$ the $n \times m$ matrix with column vectors $\mathbf{v}_{1}, \ldots, \mathbf{v}_{m}$ and by $\tilde{\mathbf{H}}_{m}$ the $(m+1) \times m$ Hessenberg matrix on the right-hand side of relation (3.7). Equation (3.7) can then be written as

$$
\mathbf{A} \mathbf{V}_{m}=\mathbf{V}_{m+1} \tilde{\mathbf{H}}_{m} .
$$

By deleting the last row of the matrix $\tilde{\mathbf{H}}_{m}$, we obtain the $m \times m$ matrix $\mathbf{H}_{m}$ and we also have

$$
\mathbf{A} \mathbf{V}_{m}=\mathbf{V}_{m} \mathbf{H}_{m}+h_{m+1, m} \mathbf{v}_{m+1} \mathbf{e}_{m}^{\top},
$$

with $\mathbf{e}_{m}$ the $m$-th column of the identity matrix of order $m$ so that the second matrix on the right-hand side of Eq. (3.9) is a rank-one matrix of size $n \times m$, with the vector $\mathbf{v}_{m+1}$ as its $m$-th column. Since the columns of matrix $\mathbf{V}_{m}$ are orthonormal, we have

$$
\mathbf{V}_{m}^{H} \mathbf{V}_{m}=\mathbf{I}
$$

and from Eq. (3.9) it follows that

$$
\mathbf{V}_{m}^{H} \mathbf{A V}_{m}=\mathbf{H}_{m}
$$

We illustrate the result of the Arnoldi algorithm in Figure 3.1, where equations (3.8) and (3.9) are depicted in terms of matrix sizes.

Due to the nature of the iterative process of the Arnoldi algorithm, it is obvious that one can stop at any given $m$ ending with the partial Hessenberg matrix of the original matrix $\mathbf{A}$. This attractive property is exploited in Section 3.4, where we use it to solve large linear systems.

\subsection{GMRES}

In the previous section we showed how Arnoldi can be used to systematically create the orthonormal basis vectors of a Krylov subspace $\mathcal{K}_{m}$ from which we also obtained the partial Hessenberg matrix of the original matrix. In this section we show how it can be used to solve linear systems equations with 


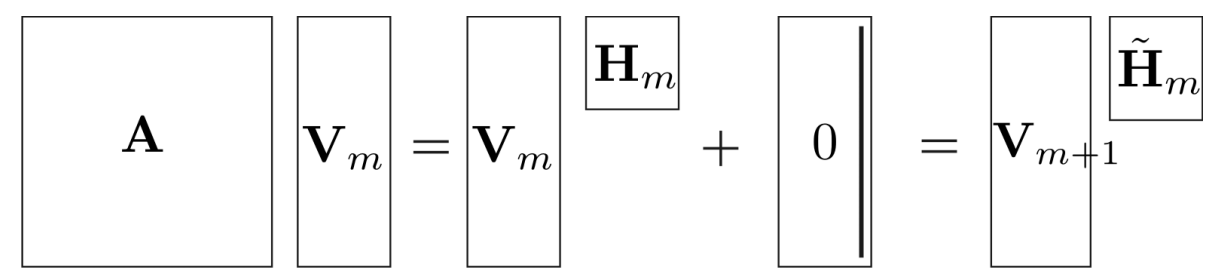

Figure 3.1: Illustration of the result of Arnoldi process.

a method called the General Minimum Residual Method (GMRES), see [7] and [8]. This is an iterative method where one seeks an approximate solution $\mathbf{x}_{m}$ living in the affine space

$$
\mathbf{x}_{0}+\mathcal{K}_{m}\left(\mathbf{A}, \mathbf{r}_{0}\right)
$$

by imposing the constraint

$$
\left\|\mathbf{r}_{m}\right\|=\min _{\mathbf{x} \in \mathbf{x}_{0}+\mathcal{K}_{m}\left(\mathbf{A}, \mathbf{r}_{0}\right)}\|\mathbf{b}-\mathbf{A} \mathbf{x}\|
$$

Explicitly, we have

$$
\mathbf{x}_{m}=\mathbf{x}_{0}+y_{1} \mathbf{v}_{1}+y_{2} \mathbf{v}_{2}+\ldots+y_{m} \mathbf{v}_{m},
$$

and this can be written in matrix form as

$$
\mathbf{x}_{m}=\mathbf{x}_{0}+\mathbf{V}_{m} \mathbf{y}
$$

where $\mathbf{y}=\left[y_{1}, y_{2}, \ldots, y_{m}\right]^{\top}$. Now for the residual at the $m$-th iteration, we can write

$$
\begin{aligned}
\mathbf{r}_{m}=\mathbf{b}-\mathbf{A} \mathbf{x}_{m} & =\mathbf{b}-\mathbf{A}\left(\mathbf{x}_{0}+\mathbf{V}_{m} \mathbf{y}\right) \\
& =\mathbf{r}_{0}-\mathbf{A} \mathbf{V}_{m} \mathbf{y} \\
& =\beta \mathbf{v}_{1}-\mathbf{V}_{m+1} \tilde{\mathbf{H}}_{m} \mathbf{y} \\
& =\mathbf{V}_{m+1}\left(\beta \mathbf{e}_{1}-\tilde{\mathbf{H}}_{m} \mathbf{y}\right)
\end{aligned}
$$

where we have introduced $\beta=\left\|\mathbf{r}_{0}\right\|_{2}$ so that

$$
\beta \mathbf{v}_{1}=\beta \mathbf{V}_{m+1} \mathbf{e}_{1} .
$$

Since the columns of $\mathbf{V}_{m+1}$ are orthonormal, the norm of the residual $\mathbf{r}_{m}$ can be written as

$$
\left\|\mathbf{r}_{m}\right\|=\left\|\mathbf{b}-\mathbf{A} \mathbf{x}_{m}\right\|=\left\|\beta \mathbf{e}_{1}-\tilde{\mathbf{H}}_{m} \mathbf{y}\right\|
$$


The vector $\mathbf{y}$ that minimizes $\left\|\beta \mathbf{e}_{1}-\tilde{\mathbf{H}}_{m} \mathbf{y}\right\|$ is denoted by $\mathbf{y}_{m}$. This vector is inexpensive to compute, since it is the solution of an $(m+1) \times m$ leastsquares problem, where $m \ll n$. Having vector $\mathbf{y}_{m}$ available, the approximate solution $\mathbf{x}_{m}$ is given by

$$
\mathbf{x}_{m}=\mathbf{x}_{0}+\mathbf{V}_{m} \mathbf{y}_{m} .
$$

The complete procedure is summarized in the following algorithm:

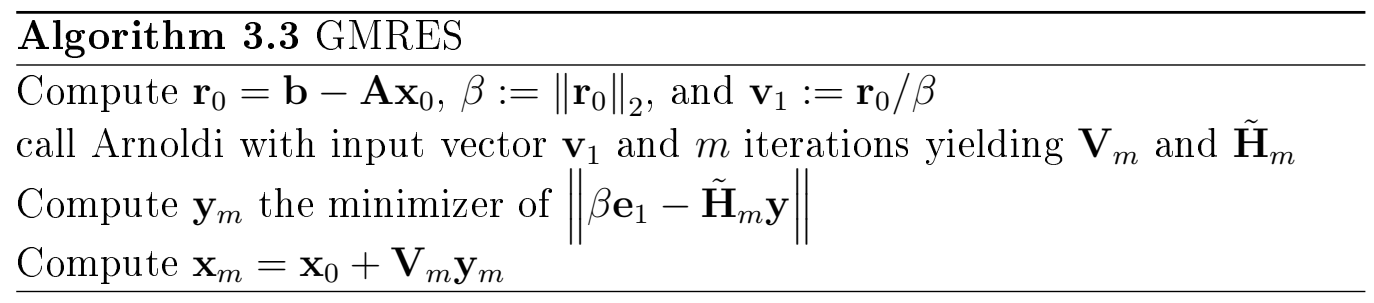

It is desirable that the algorithm is implemented in a such a way that $m$ is selected in a dynamic fashion so the approximate solution is found after the smallest number of iterations. More precisely, the algorithm can be stopped if the residual norm is lower than a given tolerance. It is obvious that calculating the residual by $\left\|\beta \mathbf{e}_{1}-\tilde{\mathbf{H}}_{m} \mathbf{y}\right\|$ is less expensive than using $\left\|\mathbf{b}-\mathbf{A} \mathbf{x}_{m}\right\|$, but there is an even more specialized approach requiring the least computational power. The method is based on the way in which leastsquares problem of Eq. (3.10) is solved where instead of constructing the QR factorization for each $\tilde{\mathbf{H}}_{1}, \tilde{\mathbf{H}}_{2}, \ldots$ separately, one obtains the $\mathbf{Q R}$ factorization of $\tilde{\mathbf{H}}_{m}$ by updating the factorization of $\tilde{\mathbf{H}}_{m-1}$. This is achieved by applying Givens rotations on $\beta \mathbf{e}_{1}$ and $\tilde{\mathbf{H}}_{m} \mathbf{y}$ and the residual norm follows in the course of updating the QR factorization, see [7].

\subsubsection{Restarted GMRES}

In practice, a large number of iterations may be required to reach a desired residual norm. This makes Algorithm 3.3 less attractive as for large $m$ the computational and the memory cost increase. A remedy is to restart the algorithm if after the $m$-th step the desired residual norm is not reached. The resulting algorithm is as follows: 


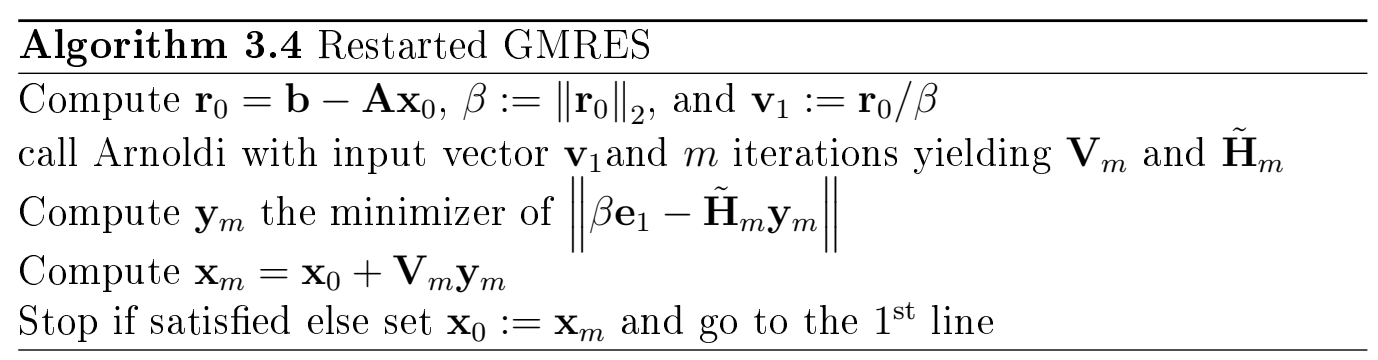

The performance of the algorithm is influenced by the choice of $m$. Some systems might converge fast enough for small $m$, others might need a large $m$. One might as well choose $m$ in a dynamic fashion where $m$ changes at every restart. The performance gain due to this choice strongly depends on the properties of matrix $\mathbf{A}$.

One drawback of restarted GMRES is that it can stagnate and the algorithm becomes less efficient if a large number of iterations is necessary for convergence. In practice, a preconditioner might be used to reduce the number of steps needed.

\subsubsection{Shifted GMRES}

Let us consider two linear systems

$$
\mathrm{Ax}=\mathrm{b}
$$

and

$$
\hat{\mathbf{A}} \hat{\mathbf{x}}=\mathbf{b}
$$

such that

$$
\hat{\mathbf{A}}=\mathbf{A}+\alpha \mathbf{I} \text {. }
$$

To easily refer to one of the two systems, we name Eq. (3.11) the seed system and Eq. (3.12) the add system. Both systems have identical Krylov subspaces with respect to any fixed vector $\mathbf{b}$. Specifically,

$$
\begin{aligned}
\mathcal{K}_{m}(\mathbf{A}, \mathbf{b}) & =\operatorname{span}\left\{\mathbf{b}, \mathbf{A} \mathbf{b}, \mathbf{A}^{2} \mathbf{b}, \ldots, \mathbf{A}^{m-1} \mathbf{b}\right\} \\
& =\operatorname{span}\left\{\mathbf{b}, \hat{\mathbf{A}} \mathbf{b}, \hat{\mathbf{A}}^{2} \mathbf{b}, \ldots, \hat{\mathbf{A}}^{m-1} \mathbf{b}\right\}=\mathcal{K}_{m}(\hat{\mathbf{A}}, \mathbf{b}) .
\end{aligned}
$$

If we would use GMRES to solve the seed and the add system, then at the $m$-th iteration, the approximate solutions of the seed and add system live in the affine subspaces $\mathbf{x}_{0}+\mathcal{K}_{m}\left(\mathbf{A}, \mathbf{r}_{0}\right)$ and $\hat{\mathbf{x}}_{0}+\mathcal{K}_{m}\left(\hat{\mathbf{A}}, \hat{\mathbf{r}}_{0}\right)$, respectively. Hence, 


$$
\mathbf{x}_{m} \in \mathbf{x}_{0}+\mathcal{K}_{m}\left(\mathbf{A}, \mathbf{r}_{0}\right) \quad \text { and } \quad \hat{\mathbf{x}}_{m} \in \hat{\mathbf{x}}_{0}+\mathcal{K}_{m}\left(\hat{\mathbf{A}}, \hat{\mathbf{r}}_{0}\right) .
$$

By taking $\mathbf{x}_{0}=\mathbf{0}$ and $\hat{\mathbf{x}}_{0}=\mathbf{0}$ as initial guesses for both systems, we would then have for both initial residuals $\mathbf{r}_{0}=\hat{\mathbf{r}}_{0}=\mathbf{b}$. This implies that the approximate solutions $\mathbf{x}_{m}$ and $\hat{\mathbf{x}}_{m}$ of Eqs. (3.11) and (3.12) live in the same Krylov subspace. Explicitly, both $\mathbf{x}_{m}$ and $\hat{\mathbf{x}}_{m}$ live in

$$
\mathcal{K}_{m}\left(\mathbf{A}, \mathbf{r}_{0}\right)=\mathcal{K}_{m}(\mathbf{A}, \mathbf{b})=\mathcal{K}_{m}(\hat{\mathbf{A}}, \mathbf{b})=\mathcal{K}_{m}\left(\hat{\mathbf{A}}, \hat{\mathbf{r}}_{0}\right)
$$

This is a very interesting result which can be exploited when solving the seed and add system simultaneously, since the orthonormal basis needs to be computed only once. Recall that at least one matrix-vector multiplication with $\mathbf{A}$ is required at every iteration of the Arnoldi algorithm in order to extend the dimension of the Krylov subspace $\mathcal{K}_{m}\left(\mathbf{A}, \mathbf{r}_{0}\right)$ to $\mathcal{K}_{m+1}\left(\mathbf{A}, \mathbf{r}_{0}\right)$. If one is able to cheaply monitor the solutions and the residuals of the add system at every iteration step, then a major computational and memory cost reduction is achieved when solving many add systems simultaneously.

Let us now be more specific. Using Eq. (3.8) and the definition of matrix $\hat{\mathbf{A}}$, we have

$$
\hat{\mathbf{A}} \mathbf{V}_{m}=\mathbf{V}_{m+1} \hat{\tilde{\mathbf{H}}}_{m}
$$

with

$$
\hat{\tilde{\mathbf{H}}}_{m}=\tilde{\mathbf{H}}_{m}+\alpha\left[\begin{array}{c}
\mathbf{I}_{m} \\
-\mathbf{0}-
\end{array}\right] .
$$

Using Eq. (3.13), we can express the residual $\hat{\mathbf{r}}_{m}$ of the add system as follows

$$
\begin{aligned}
\hat{\mathbf{r}}_{m}=\mathbf{b}-\hat{\mathbf{A}} \hat{\mathbf{x}}_{m} & =\mathbf{b}-\hat{\mathbf{A}}\left(\mathbf{x}_{0}+\mathbf{V}_{m} \hat{\mathbf{y}}\right) \\
& =\mathbf{r}_{0}-\hat{\mathbf{A}} \mathbf{V}_{m} \hat{\mathbf{y}} \\
& =\beta \mathbf{v}_{1}-\mathbf{V}_{m+1}\left[\tilde{\mathbf{H}}_{m}+\alpha\left[\begin{array}{c}
\mathbf{I}_{m} \\
-\mathbf{0}-
\end{array}\right]\right] \hat{\mathbf{y}} \\
& =\mathbf{V}_{m+1}\left(\beta \mathbf{e}_{1}-\left[\tilde{\mathbf{H}}_{m}+\alpha\left[\begin{array}{c}
\mathbf{I}_{m} \\
-\mathbf{0}-
\end{array}\right]\right] \hat{\mathbf{y}}\right) .
\end{aligned}
$$

Due to the orthonormality of the basis vectors, the 2-norm of the residual is given by

$$
\left\|\hat{\mathbf{r}}_{m}\right\|=\left\|\beta \mathbf{e}_{1}-\left[\tilde{\mathbf{H}}_{m}+\alpha\left[\begin{array}{c}
\mathbf{I}_{m} \\
-\mathbf{0}-
\end{array}\right]\right] \hat{\mathbf{y}}\right\|
$$


and this norm can be minimized at low cost for many different shift parameters $\alpha_{1}, \alpha_{2}, \ldots, \alpha_{p}$.

The algorithm for solving the seed and many add systems is as follows:

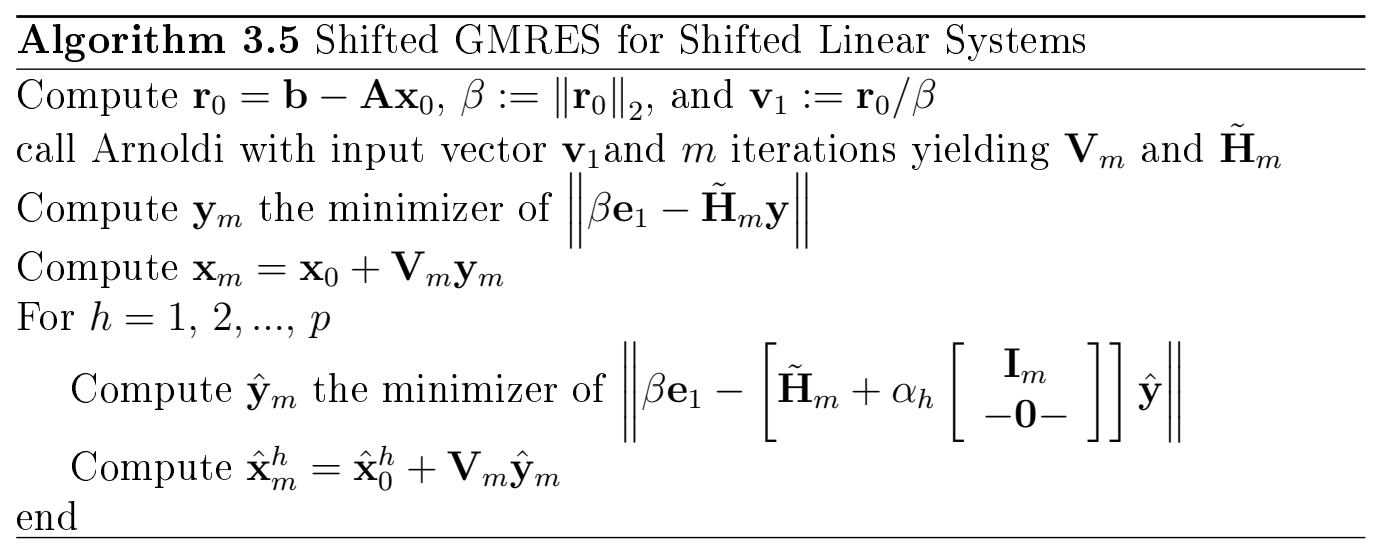

\subsubsection{Restarted GMRES for Shifted Linear Systems}

As stated earlier, the use of Krylov subspace methods becomes less efficient when considering large systems as the computational and memory cost increases with $m$. As a remedy we earlier used the Restarted GMRES, but using this method to simultaneously solve add systems is not straightforward since we may loose the shift-invariance property of the Krylov subspace.

Notice that restarting GMRES after $m$ iterations, the updated initial guesses $\mathbf{x}_{0}$ and $\hat{\mathbf{x}}_{0}$ of the seed and add system, respectively, are not identical anymore. This gives us different residuals $\mathbf{r}_{m}$ and $\hat{\mathbf{r}}_{m}$ and consequently

$$
\mathcal{K}_{m}\left(\mathbf{A}, \mathbf{r}_{m}\right) \neq \mathcal{K}_{m}\left(\hat{\mathbf{A}}, \hat{\mathbf{r}}_{m}\right),
$$

in general. However, the Krylov subspaces of the seed and add system would be identical if the residuals $\mathbf{r}_{m}$ and $\hat{\mathbf{r}}_{m}$ are colinear, that is, if

$$
\hat{\mathbf{r}}_{m}=\gamma_{m} \mathbf{r}_{m} \text { with } \gamma_{m} \in \mathbb{C} .
$$

Enforcing this colinearity condition makes Restarted GMRES useful for simultaneously solving large linear add systems. This is proposed and discussed in [2] for linear systems where matrix $\mathbf{A}$ is nonsingular and nonhermitian.

For the sake of convenience we write the residual of the seed system as

$$
\mathbf{r}_{m}=\mathbf{V}_{m+1} \mathbf{z}_{m+1} \quad \text { with } \mathbf{z}_{m+1}=\beta \mathbf{e}_{1}-\tilde{\mathbf{H}}_{m} \hat{\mathbf{y}}_{m}
$$


Now, imposing the colinearity condition of Eq. (3.16) and using Eq. (3.13), we have

$$
\begin{aligned}
& \hat{\mathbf{r}}_{m}=\gamma_{m} \mathbf{r}_{m} \\
& \mathbf{b}-\hat{\mathbf{A}}\left(\hat{\mathbf{x}}_{0}+\mathbf{V}_{m} \hat{\mathbf{y}}_{m}\right)=\gamma_{m} \mathbf{V}_{m+1} \mathbf{z}_{m+1} \\
& \hat{\mathbf{r}}_{0}-\hat{\mathbf{A}} \mathbf{V}_{m} \hat{\mathbf{y}}_{m}=\mathbf{V}_{m+1} \mathbf{z}_{m+1} \gamma_{m} \\
& \gamma_{0} \mathbf{r}_{0}-\mathbf{V}_{m+1} \hat{\tilde{\mathbf{H}}}_{m} \hat{\mathbf{y}}_{m}=\mathbf{V}_{m+1} \mathbf{z}_{m+1} \gamma_{m} \\
& \mathbf{V}_{m+1}\left(\hat{\tilde{\mathbf{H}}}_{m} \hat{\mathbf{y}}_{m}+\mathbf{z}_{m+1} \gamma_{m}\right)=\gamma_{0} \mathbf{r}_{0} \\
& \hat{\tilde{\mathbf{H}}}_{m} \hat{\mathbf{y}}_{m}+\mathbf{z}_{m+1} \gamma_{m}=\gamma_{0}\left\|\mathbf{r}_{0}\right\|_{2} \mathbf{e}_{1} \text {. }
\end{aligned}
$$

Furthermore, we used the basic assumption

$$
\hat{\mathbf{r}}_{0}=\gamma_{0} \mathbf{r}_{0} \quad \text { with } \gamma_{0} \in \mathbb{C},
$$

as otherwise the Krylov subspaces of the seed and add system would not be identical in the first place. Rewriting Eq. (3.17) in terms of unknown quantities we arrive at the following matrix system

$$
\left[\hat{\tilde{\mathbf{H}}}_{m} \mid \mathbf{z}_{m+1}\right]\left[\begin{array}{c}
\hat{\mathbf{y}}_{m} \\
\gamma_{m}
\end{array}\right]=\gamma_{0} \beta \mathbf{e}_{1},
$$

where the matrix on the left-hand side is a square matrix of order $m+1$. For conditions on the nonsingularity of this matrix, see [2]. Solving Eq. (3.18), one retrieves $\hat{\mathbf{y}}_{m}$ and $\gamma_{m}$, and the latter one can be used to cheaply monitor the norm of the residual of the add system without computing the residual $\hat{\mathbf{r}}_{m}$ explicitly. Notice that the norm of the residual $\mathbf{r}_{m}$ of the seed system is available at the course of computing $\mathbf{x}_{m}$ and for the norm of $\hat{\mathbf{r}}_{m}$ we have

$$
\left\|\hat{\mathbf{r}}_{m}\right\|=\left|\gamma_{m}\right| \cdot\left\|\mathbf{r}_{m}\right\| .
$$

The Shifted Restarted-GMRES algorithm can be written as follows: 


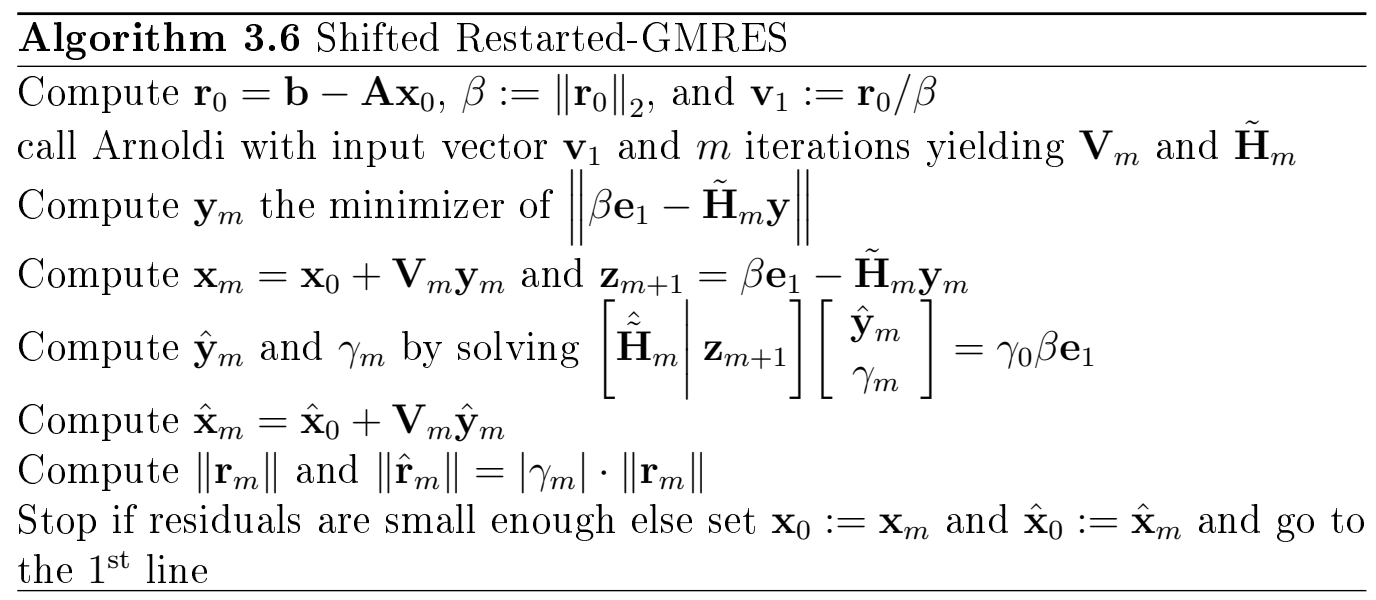

In general, the most expensive computation in Algorithm 3.6, after Arnoldi, is computing $\mathbf{x}_{m}$ and $\hat{\mathbf{x}}_{m}$. It seems like one can avoid computing these until the residuals are small enough. However, restarting the algorithm without updating $\mathbf{x}_{0}$ and $\hat{\mathbf{x}}_{0}$ is useless as the Krylov subspace to be generated would be identical to the one before the restart, resulting in the same approximations at the end of the algorithm. Updating the approximations is unavoidable and becomes a problem when solving many add systems simultaneously as then the memory load increases. For every add system with $\alpha=\alpha_{h}$, for $h=$ $1,2, \ldots, p$, one need to compute and store all $n$-vectors $\mathbf{x}_{m}^{h}$ at every restart. The algorithm to simultaneously solve many add systems is as follows:

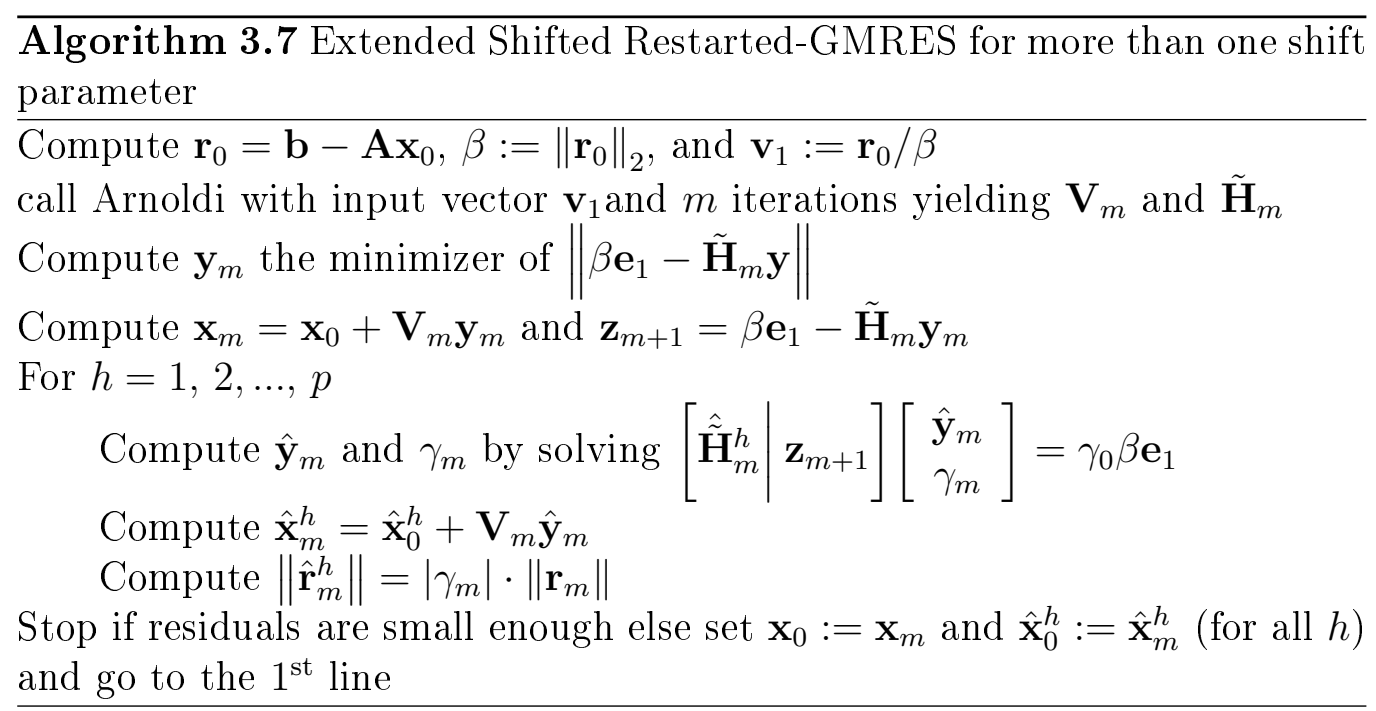

Matrix $\hat{\tilde{\mathbf{H}}}_{m}^{h}$ in above algorithm is an $(m+1) \times m$ Hessenberg matrix shifted by $\alpha_{h} \mathbf{I}$ as expressed in Eq. (3.14) with $\alpha=\alpha_{h}$. 
Now that we have given the complete algorithm we need to rewrite the object equation such that the implementation of the presented algorithms is straightforward.

To this end, we first introduce the contrast source $\mathbf{w}=-\chi \mathbf{u}$, where the minus sign is included so that the object equation can be rewritten as a seed system. In particular,

$$
\mathbf{A}(\alpha) \mathbf{w}=\mathbf{u}^{\text {inc }} \text { where } \mathbf{A}(\alpha)=\mathbf{G}+\alpha \mathbf{I}
$$

and $\alpha=-1 / \chi$. We choose $\chi$ such that the seed system has the worst convergence behavior. Then the equation to be solved for all contrast functions can be written as

$$
\left(\mathbf{A}(\alpha)+\beta\left(\alpha_{h}\right) \mathbf{I}\right) \hat{\mathbf{w}}^{h}=\mathbf{u}^{\text {inc }} \text { where } \beta\left(\alpha_{h}\right)=\alpha_{h}-\alpha,
$$

$\hat{\mathbf{w}}^{h}=\frac{1}{\alpha_{h}} \mathbf{u}^{h}$ and $h=1,2, \ldots, p$. For $\beta=0$, Eq. (3.20) is the seed system and for $\beta \neq 0$ it is an add system. Notice that $\mathbf{u}^{h}$ refers to the total field inside the object with contrast function $\chi_{h}$. Now the Krylov subspace might be generated using the seed system matrix $\mathbf{A}(\alpha)$ and $\mathbf{r}_{0}=\mathbf{u}^{\text {inc }}$. It is obvious that the choice of the system matrix might as well be $\mathbf{G}$ or $\mathbf{A}\left(\alpha_{h}\right)=\mathbf{A}(\alpha)+\beta\left(\alpha_{h}\right) \mathbf{I}$ as they all yield the same Krylov subspaces, that is, we have

$$
\mathcal{K}_{m}\left[\mathbf{A}(\alpha), \mathbf{r}_{0}\right]=\mathcal{K}_{m}\left[\mathbf{A}\left(\alpha_{h}\right), \mathbf{r}_{0}\right]=\mathcal{K}_{m}\left[\mathbf{G}, \mathbf{r}_{0}\right] .
$$

Algorithm 3.7 exploits restarts without loosing the shift-invariance property of a Krylov subspace, by imposing the colinearity condition of Eq. (3.16). This algorithm is the most important block of our effective inversion scheme. 


\section{Chapter 4}

\section{Numerical Results}

\subsection{Introduction}

In this chapter we present the experimental results of the modified GMRES method we used to retrieve the medium parameters of a homogeneous object. In Section 4.2 we present the configuration and the parameters we used. We present the results of the inverse scattering problem by illuminating the scatterer by single frequency radiation source in Section 4.3 and in Section 4.4 we illuminate the scatterer by multiple frequency waves and present the corresponding results. Finally, in Section 4.5 we show how the algorithm can be used to map the contrast function of an homogeneous object with randomly perturbed inclusions to a homogeneous one.

\subsection{Configuration}

We retrieve the medium parameters $\sigma^{\text {sc }}$ and $\varepsilon^{\text {sc }}$ of a homogeneous square block of size $\ell=\lambda$, with $\lambda$ the wavelength in vacuum corresponding to the frequency of operation. This block is located in vacuum and so are the source and receiver unit. We have used a single-source and a single-receiver located symmetrically above the block at a distance of $\ell / 2$ away from the block. The distance between the source and receiver unit is $4 \mathrm{~cm}$. This configuration is depicted in Figure 4.1.

We compute the true scattered field $\hat{E}_{z}^{\mathrm{sc}}\left(\boldsymbol{\rho}_{\mathbf{r}}, \omega\right)$ at the receiver location $\boldsymbol{\rho}_{\mathbf{r}}$ and at a particular angular frequency $\omega$ using Eq. (2.60). For the true medium parameters we take $\varepsilon^{\mathrm{sc}}=4.5$ and $\sigma^{\mathrm{sc}}=5 \mathrm{mS} / \mathrm{m}$ and compute the contrast function $\chi(\omega)$ using Eq. (2.33).

Now that we have computed the true scattered field we need to solve forward problems to find the modeled scattered fields for the collection of 


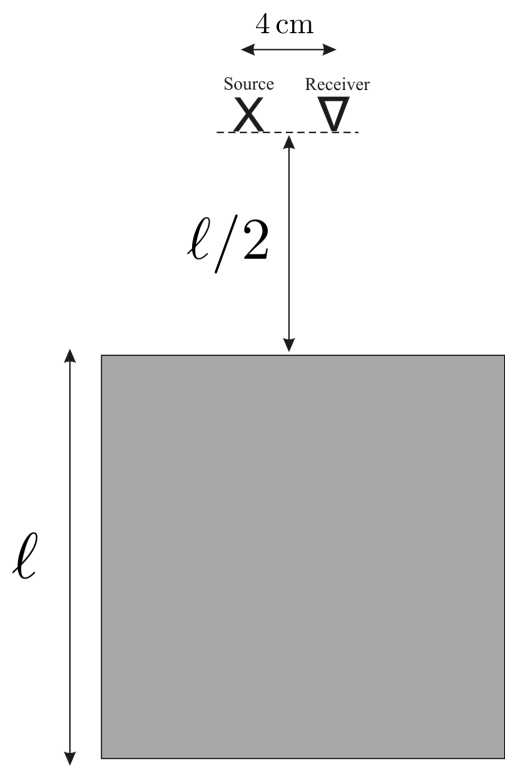

Figure 4.1: The configuration used for the experimental results.

the contrast values. This collection of interest is contained in the intervals $1 \leq \varepsilon_{\mathrm{r}} \leq 10$ and $0 \leq \sigma \leq 10 \mathrm{mS} / \mathrm{m}$. The $\chi$-values of interest lie the complex plane as depicted in Figure 4.2 which we have discretized using a $40 \times 40$ grid. Therefore, there are $1600 \chi$-values but since for $\chi=0$ the scattered and total field are known, we have 1599 forward problems to solve.

To make the implemented algorithm efficient, it is of great importance to identify the contrast coefficient for which the largest number of iterations is needed to reach a certain prescribed and sufficiently small residual. It is then guaranteed that the residuals corresponding to the remaining $\chi$-values have reached a sufficiently small level as well. It would be reasonable to assume that for the largest $|\chi|$ most iterations are needed to reach a sufficiently small residual. This $\chi$-value we have depicted in Figure 4.2 as node B. Excessive numerical testing, however, showed that the largest $|\chi|$ is not the worst-case coefficient. It turned out that the worst-case $\chi$ is given by $\operatorname{Re}(\chi)=\varepsilon_{\mathrm{r} ; \max }-1$ and $\operatorname{Im}(\chi)=0$, in Figure 4.2 depicted as node A. It is thus the case where one considers a lossless object with maximum permittivity which gives us the worst-case system to solve. In Figure 4.3 we have illustrated the normalized 2-norm of the residual vectors for different nodes of Figure 4.2. We considered a zero initial guess, thus $\mathbf{r}_{0}=\mathbf{u}^{\text {inc }}$. From Figure 4.3 it is obvious that the contrast coefficient corresponding to node A shows the worst convergence behavior.

The identified worst-case $\chi$ we denote by $\chi_{\mathrm{A}}$ and its corresponding coefficient 


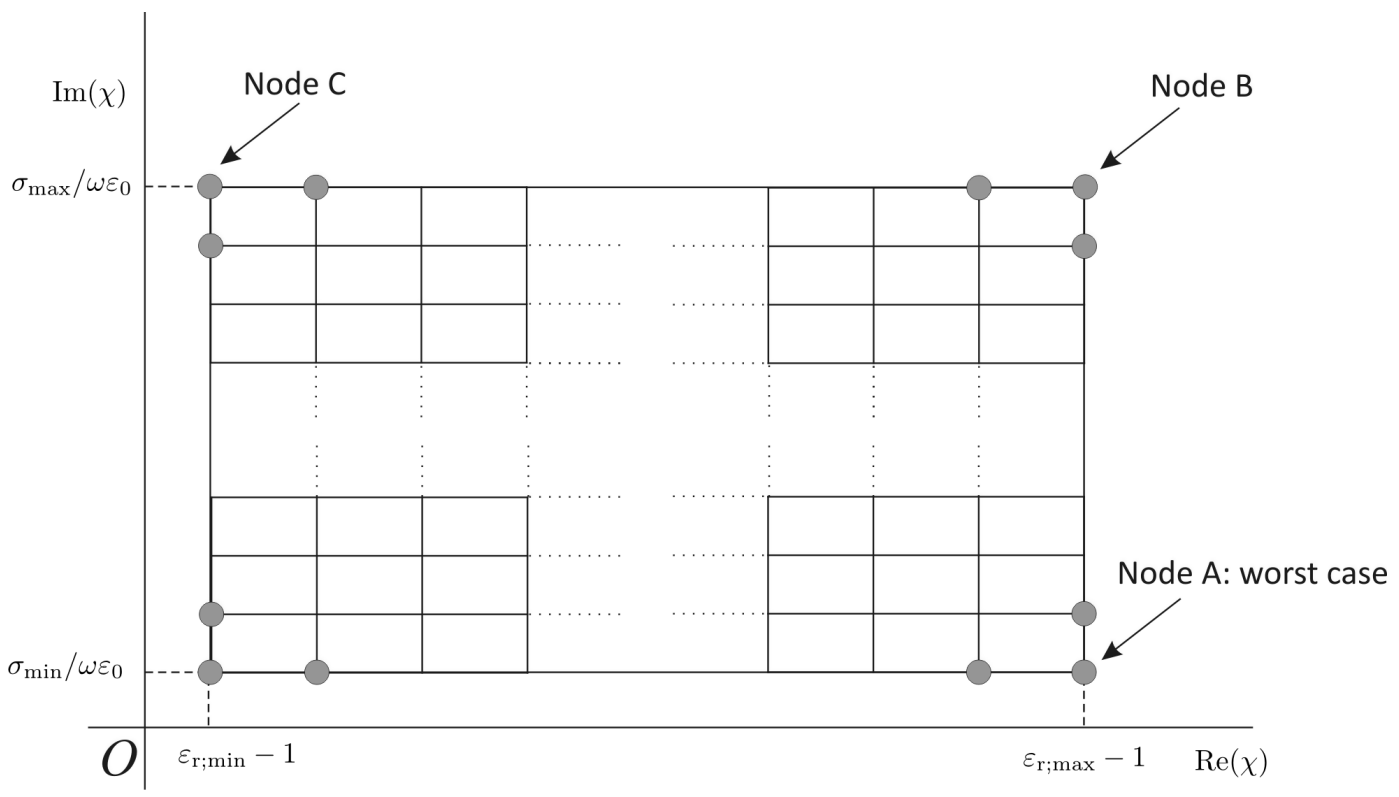

Figure 4.2: Grid of $\chi$-values of interest

by $\alpha_{\mathrm{A}}=-1 / \chi_{\mathrm{A}}$. The worst-case seed system is

$$
\mathbf{A}\left(\alpha_{\mathrm{A}}\right) \mathbf{w}=\mathbf{u}^{\text {inc }} \quad \text { with } \mathbf{w}=-\chi_{\mathrm{A}} \mathbf{u}
$$

and the approximated solution $\mathbf{w}_{m}$ is found by running the GMRES algorithm until a prescribed tolerance for the normalized residual vector is reached. Thereafter the add system

$$
\left(\mathbf{A}\left(\alpha_{\mathrm{A}}\right)+\beta\left(\alpha_{h}\right) \mathbf{I}\right) \hat{\mathbf{w}}^{h}=\mathbf{u}^{\text {inc }} \quad \text { where } \beta\left(\alpha_{h}\right)=\alpha_{h}-\alpha_{\mathrm{A}} \text { and } \hat{\mathbf{w}}^{h}=\frac{1}{\alpha_{h}} \mathbf{u}^{h},
$$

is cheaply solved by finding $\hat{\mathbf{y}}_{m}$, the minimizer of

$$
\left\|\mathbf{r}_{m}\right\|=\|\| \mathbf{u}^{\mathrm{inc}}\left\|\mathbf{e}_{1}^{(m+1)}-\left[\tilde{\mathbf{H}}_{m}+\beta\left(\alpha_{h}\right)\left[\begin{array}{c}
\mathbf{I}_{m} \\
-0-
\end{array}\right]\right] \hat{\mathbf{y}}\right\|,
$$

where after the approximated solution $\hat{\mathbf{w}}_{m}^{h}$ is found by

$$
\hat{\mathbf{w}}_{m}^{h}=\hat{\mathbf{w}}_{0}^{h}+\mathbf{V}_{m} \hat{\mathbf{y}}_{m} .
$$

We have summarized this procedure in Algorithm 3.5 where $\alpha_{h}$ now represents all the other contrast coefficients, in our case 1598 in total. This means that the minimizer of Eq. (4.1) has to be computed 1598 times. Notice that 


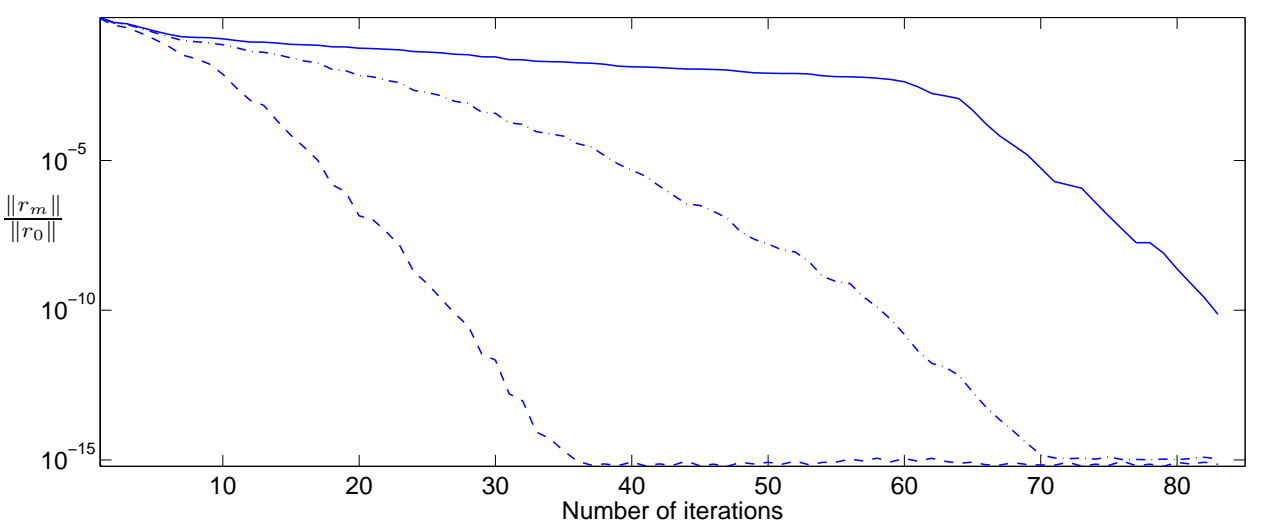

Figure 4.3: Normalized 2-norm of the residual coefficients corresponding to node A (solid line), node B (dashed-dotted line), and node C (dashed line).

for $\chi=0$ the total field is known, namely $\mathbf{u}=\mathbf{u}^{\text {inc }}$. To this end we only need to save an $(m+1) \times m$ matrix $\tilde{\mathbf{H}}_{m}$ and the $n \times m$ matrix $\mathbf{V}_{m}$.

When considering large linear systems restarting the GMRES algorithm becomes inevitable as $m$ becomes large. As we have seen in Section 3.4.3 if the algorithm is restarted after $p$ iterations then all the updated approximations $\hat{\mathbf{w}}_{p}^{h}$ need to be saved. This is necessary as they serve as an initial guess later when the $p$-th iteration is reached again. Here is where we might have a storage problem as there are $1599 n$-vectors that need to be stored since we need them before a restart of the GMRES algorithm occurs. In general, if the grid of Figure 4.2 is denoted as $g \times s$ then there are $g \cdot s$ vectors of size $n$ to be saved. However this storage problem can be avoided at the cost of more computational power. In particular we consider treating parts of the $g \times s$ grid separately. This means that we run Algorithm 3.7 for a smaller $c \times d$ grid, with $c<g$ and $d<s$, requiring $c \cdot d$ vectors of size $n$ to be stored. After computing the objective function (3.4) of all the contrast coefficients corresponding to the $c \times d$ grid, we shift this grid to cover another part of the $g \times s$ grid. We run Algorithm 3.7 again for the new covered part of the $g \times s$ grid and repeat this procedure until the complete $g \times s$ grid has been treated. This partitioning of the $g \times s$ grid have been illustrated in Figure 4.4 .

Inside a $c \times d$ grid we need to identify a contrast coefficient for which we have a worst-case seed system to solve. It is now reasonable to assume that inside each $c \times d$ grid the worst-case coefficient $\alpha_{\text {worst }}=-1 / \chi_{\text {worst }}$ is the one for which the following holds 


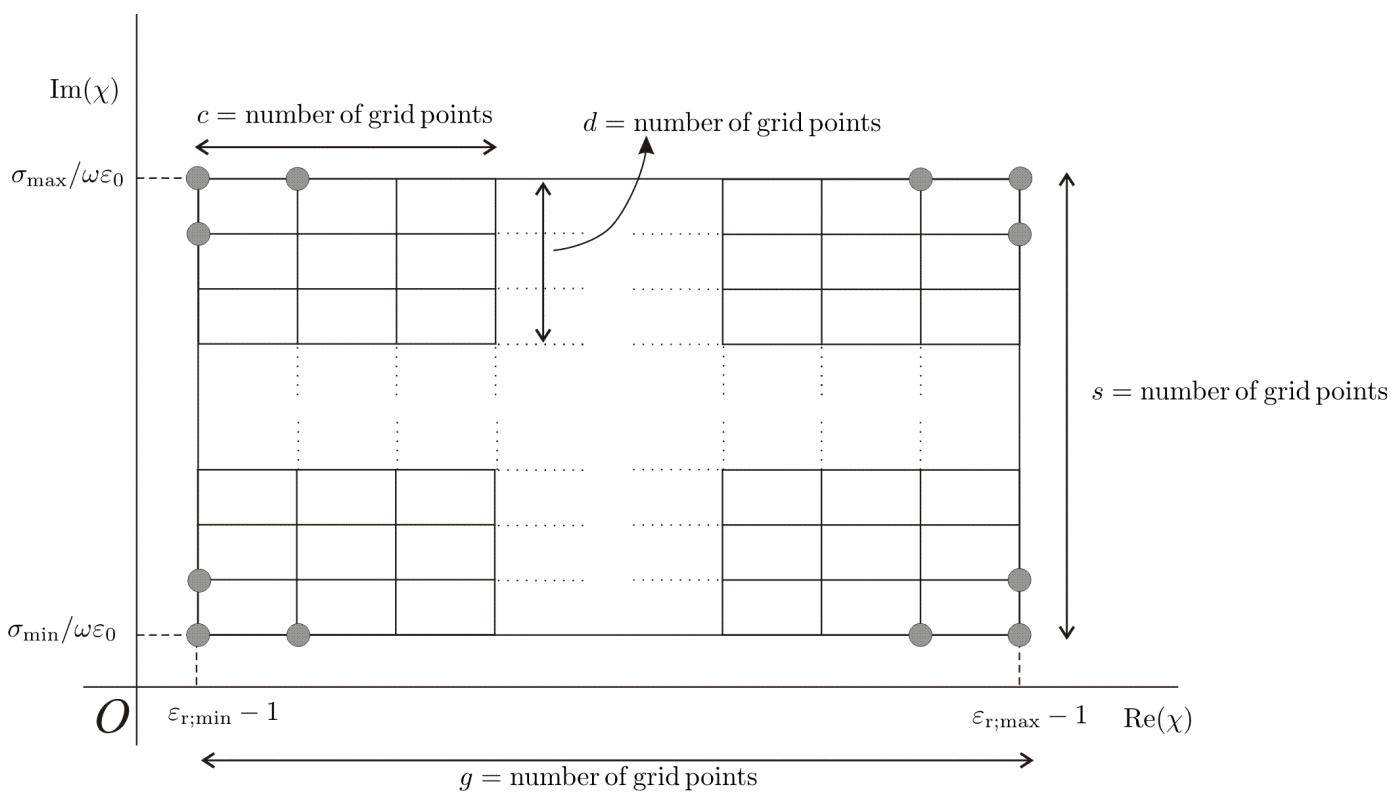

Figure 4.4: Partitioning of the grid of $\chi$-values of interest. In our experiments we have $\sigma_{\min }=0, \sigma_{\max }=10 \mathrm{mS} / \mathrm{m}, \varepsilon_{\mathrm{r} ; \min }=1$ and $\varepsilon_{\mathrm{r} ; \max }=10$.

$$
\operatorname{Max}[\operatorname{Re}(\chi)]=\operatorname{Re}\left(\chi_{\text {worst }}\right) \text { and } \operatorname{Min}[\operatorname{Im}(\chi)]=\operatorname{Im}\left(\chi_{\text {worst }}\right),
$$

where $\chi$ corresponds to all $\chi$-values inside a $c \times d$ grid. Numerical testing confirms this assumption.

In the next two sections we present the results of our inversion method by illuminating the object by a single and a multiple frequency source.

\subsection{Single Frequency Radiation Source}

We take a homogeneous square block of size $\lambda_{42}$, with $\lambda_{42}$ the free-space wavelength corresponding to $f=42 \mathrm{MHz}$. Since we are solving the forward solution for contrast values with $1 \leq \varepsilon_{\mathrm{r}} \leq 10$ and $0 \leq \sigma \leq 10 \mathrm{mS} / \mathrm{m}$ we choose the discretization of the object such that the stepsize $\delta$ satisfies the following rule

$$
(\text { nppw }-1) \delta=\lambda \text { with nppw } \geq 15,
$$

everywhere inside the domain of interest. In the above expression, nppw stands for "number of points per wavelength" implying that the spatial stepsize $\delta$ is chosen such that we have at least 15 points per wavelength. We 

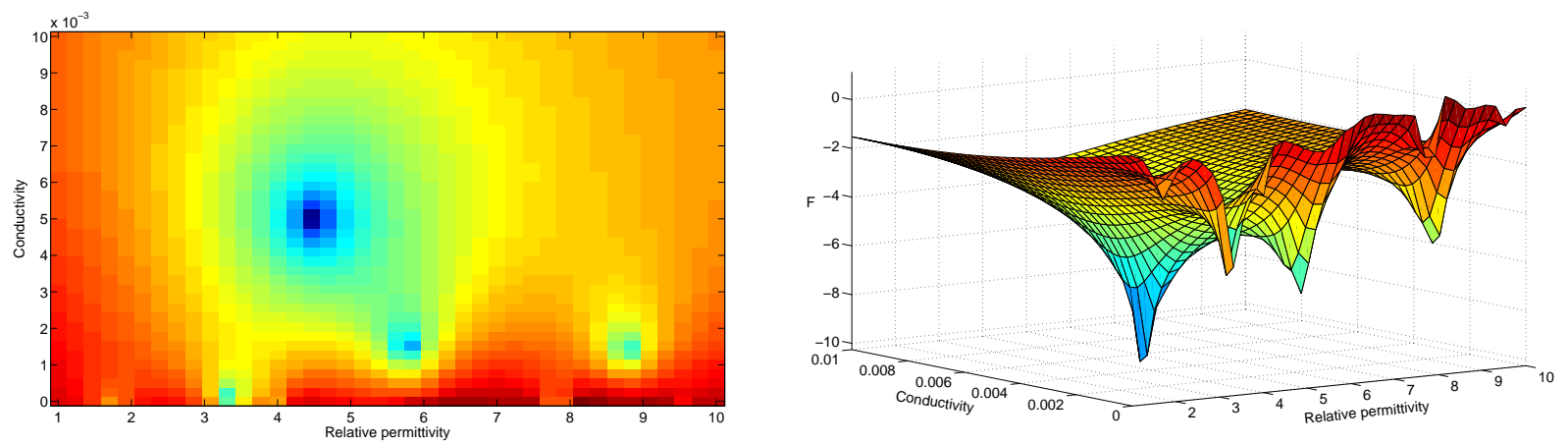

Figure 4.5: Top view (left) and surface plot (right) of the objective function $F$ on the domain of interest for a single frequency source of $f=42 \mathrm{MHz}$. Object size is $\ell=\lambda_{42}$.

have obtained satisfying results for nppw $\geq 15$ but one might as well choose another value. To satisfy this rule for the complete domain of interest, it is necessary to let it satisfy for the smallest $\lambda$. Since

$$
\lambda=\frac{c_{0}}{f \sqrt{\varepsilon_{\mathrm{r}}}},
$$

we have $\lambda_{\min }$ for $\varepsilon_{\mathrm{r} ; \max }=10$. Hereby the objects corresponding to $1 \leq \varepsilon_{\mathrm{r}}<10$ are sampled with more points per wavelength.

In Figure 4.5 we illustrate the resulting objective function on the domain of interest obtained by illuminating an object of size $\lambda_{42}$. The true parameters $\varepsilon^{\mathrm{sc}}=4.5$ and $\sigma^{\mathrm{sc}}=5 \mathrm{mS} / \mathrm{m}$ are easily recognized, yet there are a few other local minima around the $\sigma=0$ axis. The results become worse if we consider a larger object of size $3 \lambda_{42}$. As seen from Figure 4.6, the objective function has more sharper local minima although these minima are still larger than the global minimum that corresponds to the true medium parameters.

\subsection{Multiple-Frequency Radiation Source}

To improve our results, we illuminate the object by waves with two additional frequencies, namely with $f=30 \mathrm{MHz}$ and $f=36 \mathrm{MHz}$. The objective function becomes 

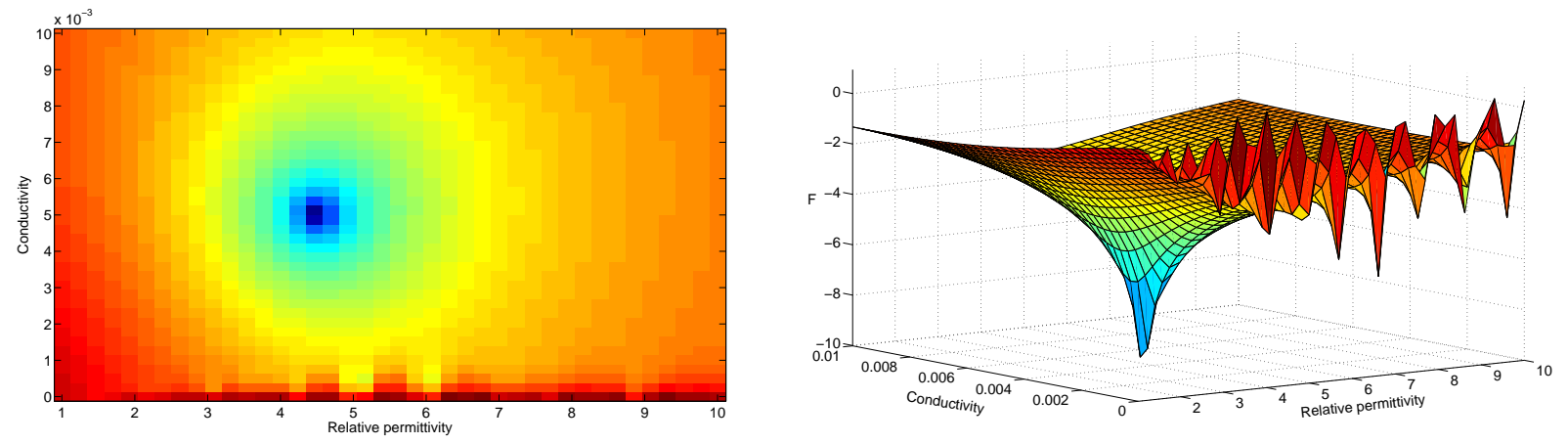

Figure 4.6: Top view (left) and surface plot (right) of the objective function $F$ on the domain of interest for a single frequency of $f=42 \mathrm{MHz}$. Object size is $\ell=3 \lambda_{42}$.

$$
F=\sum_{k=1}^{3} m_{k} \frac{\left|\hat{E}_{z}^{\mathrm{sc}}\left(\boldsymbol{\rho}_{\mathbf{r}}, \omega_{k}\right)-u^{\mathrm{sc}}\left(\omega_{k}\right)\right|^{2}}{\left|\hat{E}_{z}^{\mathrm{sc}}\left(\boldsymbol{\rho}_{\mathbf{r}}, \omega_{k}\right)\right|^{2}} \quad \text { with } \sum_{k=1}^{3} m_{k}=1
$$

and we take $m_{k}=1 / 3$ for $k=1,2,3$. The objective function is evaluated for three different frequencies by computing the forward solutions for each frequency separately. Hence, Algorithm 3.7 is used three times to compute the objective function for the domain of interest. The object has to be discretized such that the stepsize $\delta$ satisfies Eq. (4.2) for all wavelengths. It is therefore sufficient to satisfy this equation for $\lambda_{42,10}$, where $\lambda_{42,10}$ is the wavelength inside an object with $\varepsilon_{\mathrm{r}}=10$ when illuminated by a $f=42 \mathrm{MHz}$ wave. The results for an object of size $3 \lambda_{42}$ are depicted in Figure 4.7. We observe that objective function becomes smoother than in the case of single frequency and a number of false minima have disappeared. Furthermore, the global minimum remains at the same position, and its location corresponds to the true parameters.

\subsection{Effective Inversion}

Until now we have applied our technique to homogeneous objects only. We also can use this technique to characterize an inhomogeneous object. Specifically, we try to map the overall contrast function of an inhomogeneous scatterer to that of a homogeneous one, which has the same external boundary and location as the inhomogeneous scatterer. This procedure is called effective inversion and the contrast function of the resulting homogeneous object 

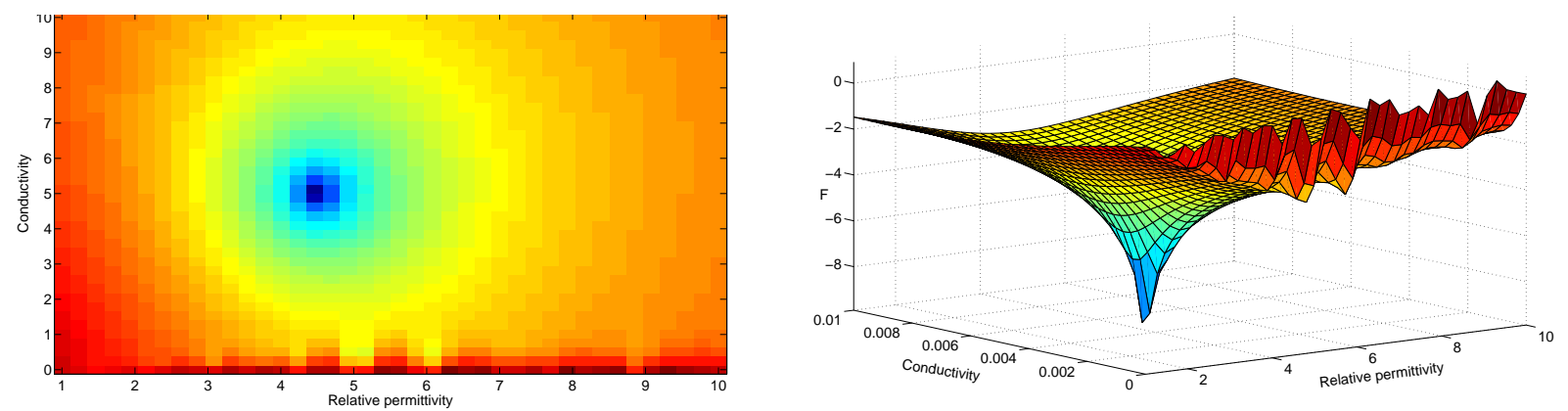

Figure 4.7: Top view (left) and surface plot (right) of the objective function $F$ on the domain of interest using three frequencies $(f=30, f=36$ and $f=42 \mathrm{MHz}$ ). Object size is $\ell=3 \lambda_{42}$.

is referred to as an effective contrast function. The general idea of effective inversion is illustrated in Figure 4.8. Characterization of an inhomogeneous object is an important topic in effective medium theory.

In our example we try to match the scattered field due to a homogeneous object to the scattered field generated by a homogeneous object with randomly (uniformly) distributed inclusions. We take for the contrast parameters of the homogeneous object $\varepsilon_{r ; 1}=4.5$ and $\sigma_{1}=5 \mathrm{mS} / \mathrm{m}$, while the parameters $\varepsilon_{r ; i}$ and $\sigma_{i}$, corresponding to the parameters of the inclusions, are randomly chosen between a certain range around $\varepsilon_{r ; 1}$ and $\sigma_{1}$ as

$$
\begin{aligned}
3.5 & \leq \varepsilon_{r ; i} \leq 5.5 \\
4 \mathrm{mS} / \mathrm{m} & \leq \sigma_{i} \leq 6 \mathrm{mS} / \mathrm{m},
\end{aligned}
$$

for $i=2,3, \ldots, q$, where $q$ is the total number of inclusions. We have experimented with different number of inclusions and of different sizes such that

$$
0<\text { length, width }<\ell / 10 \text {. }
$$

Such an inhomogeneous object is illustrated in Figure 4.9. It consists of 40 inclusions each having a different conductivity and relative permittivity. The values of the parameters can be seen from the colorbar next to each graph. We have illuminated this scatterer by waves of three different frequencies ( $f=30, f=36$ and $f=42 \mathrm{MHz}$ ) to compute the scattered field data for the same domain of interest, namely $1 \leq \varepsilon_{\mathrm{r}} \leq 10$ and $0 \leq \sigma \leq 10 \mathrm{mS} / \mathrm{m}$. Using the cost functional of Eq. (4.3), we observe from Figure 4.10 that the 

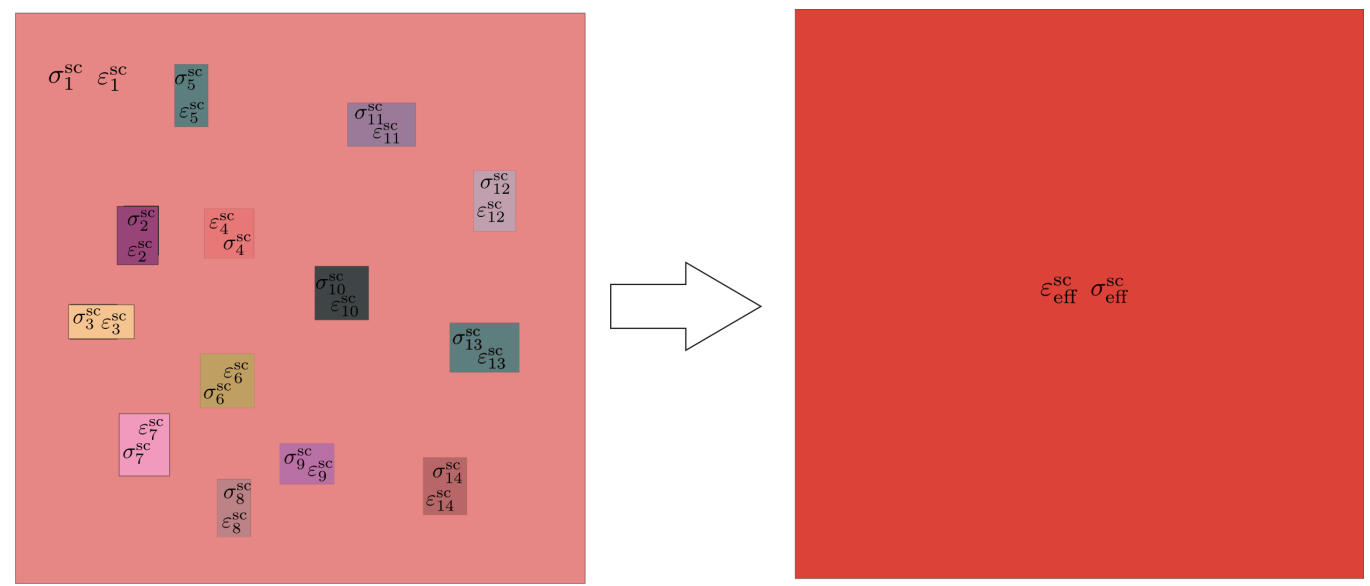

Figure 4.8: Overall contrast function of an inhomogeneous object (left) mapped to a homogeneous one (right). Each color corresponds to a different conductivity and permittivity. The overall contrast function is referred to as the effective contrast function.

effective constitutive parameter is related to the average of the true medium parameters. We have not established this correspondence analytically. We have experimented with many different randomly generated inhomogeneous media and the results of our effective inversion method are stable. This method can further be used to investigate to what extent it is possible to find an effective homogeneous scatterer for an inhomogeneous object in general.
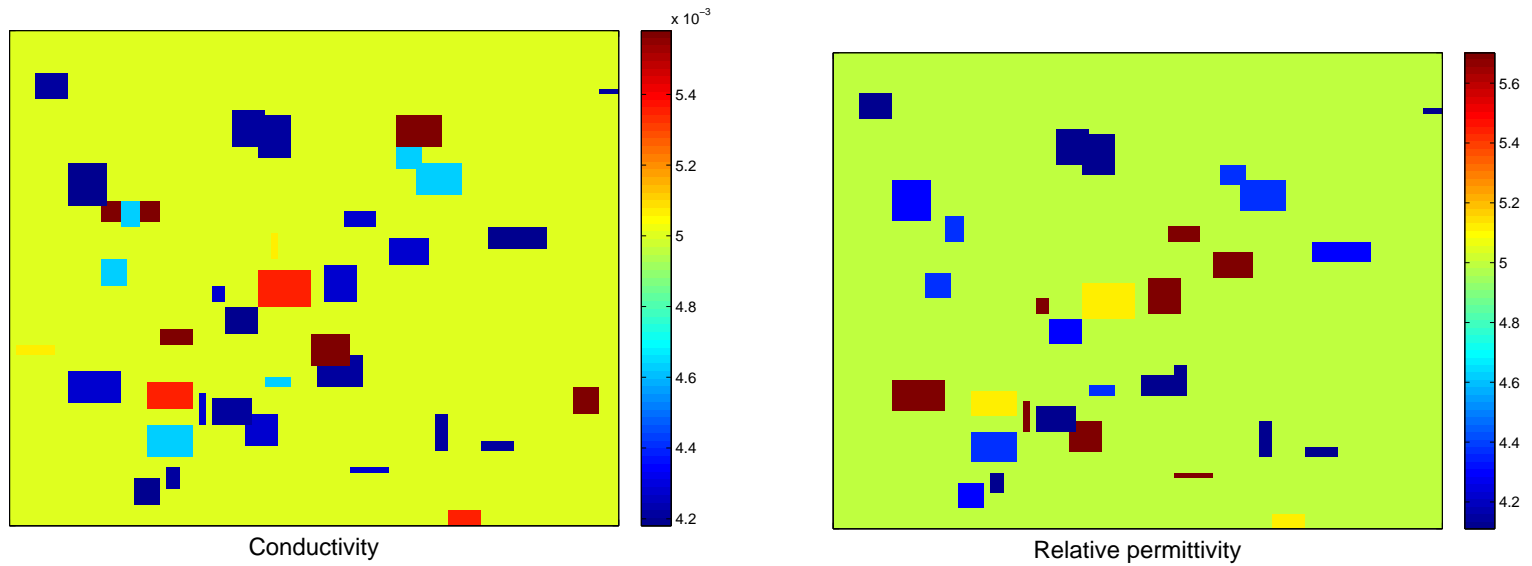

Figure 4.9: Conductivity $[\mathrm{S} / \mathrm{m}]$ (left) and relative permittivity (right) of the inclusions within the inhomogeneous scatterer. 

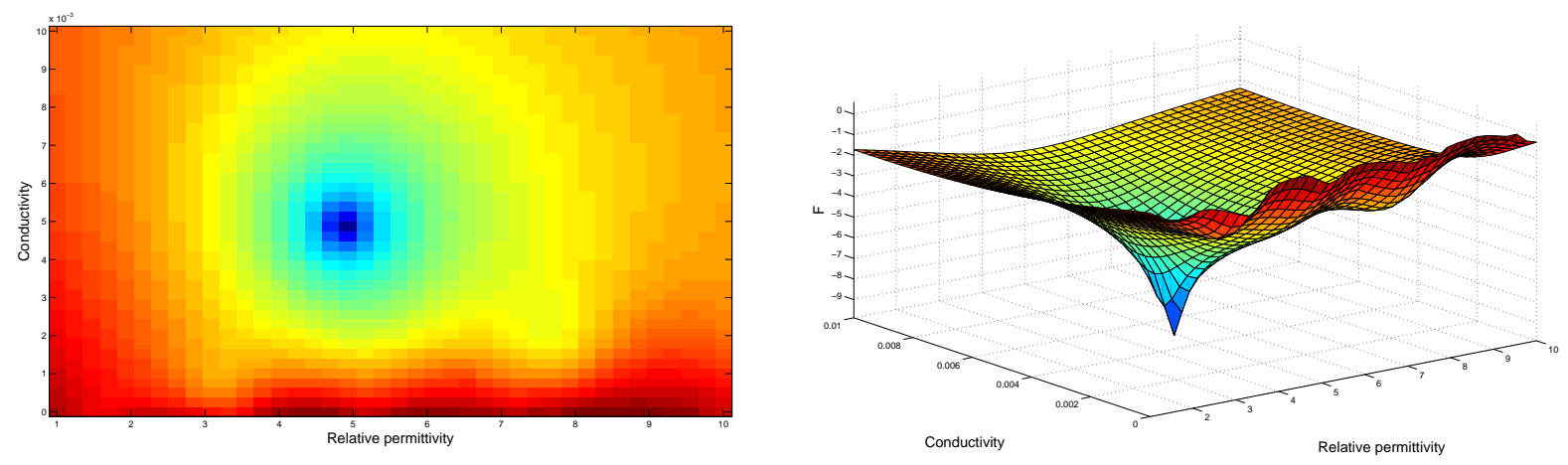

Figure 4.10: Top view (left) and surface plot (right) of the objective function $F$ for a homogeneous effective scatterer using three frequencies $(f=30$, $f=36$ and $f=42 \mathrm{MHz}$ ). Global minimum corresponds to the effective constitutive parameter. Object size is $\ell=\lambda_{42}$. 


\section{Chapter 5}

\section{Conclusions \& Recommendations}

We have presented an iterative inversion method, based on Krylov subspaces, to retrieve $\sigma^{\mathrm{sc}}$ and $\varepsilon^{\mathrm{sc}}$ of a homogeneous object and presented numerical test results on objects of size up to $\ell=3 \lambda$. In the presented method, we have exploited the shift-invariance property of Krylov subspaces to efficiently solve the object equation for many contrast parameters. The method further ensures that the Euclidean norm of all residuals is minimum and guarantees successive residuals of the field approximations, for a given contrast, to be a decreasing function. To maximally utilize the presented method, we have identified the worst-case contrast coefficient given by

$$
\operatorname{Re}\left(\chi_{\text {worst }}\right)=\operatorname{Max}[\operatorname{Re}(\chi)] \text { and } \operatorname{Im}\left(\chi_{\text {worst }}\right)=\operatorname{Min}[\operatorname{Im}(\chi)] \text {. }
$$

When dealing with electrically large objects (objects large compared with the wavelength of the electromagnetic field) and problems requiring a large number of Arnoldi iterations, the algorithm needs to be restarted as the memory and computational load increases with the number of iterations. As we might loose the shift-invariance property of Krylov subspaces at restarts, we have enforced the residuals of the seed and add system to be colinear, as proposed in [2]. Hence, when restarting at $m$-th iteration, we require $\hat{\mathbf{r}}_{m}=\gamma_{m} \mathbf{r}_{m}$, for $\gamma_{m} \in \mathbb{C}$, as we then have,

$$
\mathcal{K}_{m}\left(\mathbf{A}, \mathbf{r}_{m}\right)=\mathcal{K}_{m}\left(\hat{\mathbf{A}}, \hat{\mathbf{r}}_{m}\right) .
$$

Restarting the algorithm solves the storage problem as the number of Krylov basis vectors to be stored is kept low. On the other hand, it introduces another storage problem, since we need to store all the approximated fields at every restart. This is inevitable as these field values are needed to update the approximated fields at the next restart. To this end, we partition the grid of $\chi$-values of interest and treat them separately requiring the generation 
of Krylov subspaces for each part of the grid of $\chi$-values. More research is needed to overcome this storage problem and suitable preconditioners may further reduce the number of basis vectors that need to be stored.

Furthermore, the presented method can also be used to characterize inhomogeneous objects. This is a very important topic in effective medium theory where one seeks for the effective contrast coefficient of an inhomogeneous scatterer. We have presented numerical results where we have retrieved the effective contrast coefficient of a homogeneous object with randomly distributed inclusions. Our results look very promising, but further research is needed to investigate to what extent it is possible to find an effective contrast coefficient for such scatterers.

Finally, we like to point out that although we have presented our method for 2D configurations, 3D configurations can be handled in a straightforward manner by simply replacing the $2 \mathrm{D}$ integral operators by their $3 \mathrm{D}$ counterparts. 


\section{Appendix A}

Accepted Paper Progress in Electromagnetics Research Symposium (PIERS 2010), Cambridge, USA, on 5-8 July, 2010 


\title{
A Krylov Subspace Approach to Parametric Inversion of Electromagnetic Data Based on Residual Minimization
}

\author{
E. Balidemaj ${ }^{1}$ and R. F. Remis ${ }^{1}$ \\ ${ }^{1}$ Laboratory of Electromagnetic Research \\ Faculty of Electrical Engineering, Mathematics and Computer Science \\ Delft University of Technology \\ Mekelweg 4, 2628 CD Delft, The Netherlands
}

\begin{abstract}
In this paper we present a Krylov subspace technique and use residual minimization to efficiently solve parametric electromagnetic inversion problems. We exploit the shift-invariance property of Krylov subspaces to compute total fields inside a homogeneous object for a whole range of contrast values. As soon as these fields are found, we can determine the corresponding scattered fields in a straightforward manner. This approach allows us to solve the inverse problem by simply inspecting an objective function which measures the discrepancy between the measured and modeled scattered field data.
\end{abstract}

\section{INTRODUCTION}

Many iterative inversion methods use forward solutions in every inversion step. For homogeneous objects, forward problems can be solved efficiently by exploiting the shift-invariance property of Krylov subspaces. To be specific, solving a forward problem for a homogeneous object using an integral equation approach essentially amounts to evaluating a matrix resolvent in which the inverse of the contrast coefficient acts as the resolvent parameter. The action of this resolvent function on a given vector can be evaluated very efficiently by exploiting the shift-invariance property of a Krylov subspace. After only a single run of a Krylov subspace method, field approximations can be constructed for a whole range of contrast coefficients. In previous work [1], we constructed these field approximations using the Arnoldi algorithm in combination with a Full Orthogonalization (FO) approach. The drawback of this FO approach is that, for a given contrast, the norm of the residuals corresponding to successive field approximations is not necessarily a nonincreasing function. In this paper, we remedy this problem by constructing field approximations for which the 2-norm of the corresponding residual is minimum (similar to the well known Generalized Minimum Residual or GMRES method, [3]). For a whole range of contrast parameters, we only need to solve small-scale least-squares problems and for a given contrast coefficient, the 2-norm of the residual is guaranteed to be nonincreasing as the number of iterations increases. Since all Krylov basis vectors need to be stored in the Arnoldi algorithm, we may run into storage problems especially for electrically large objects. A restarted version of the above method as proposed in [2] may then be applied.

Given the Krylov field approximations, the scattered field at specified receiver locations can be computed in a straightforward manner and the inverse scattering problem can actually be solved by inspecting a (possibly nondifferentiable) objective function which measures the discrepancy between the true and modeled scattered field data. The effectiveness of the method is illustrated by a number of numerical examples for single and multi-frequency data.

\section{INTEGRAL REPRESENTATIONS AND THEIR DISCRETIZED COUNTERPARTS}

We consider E-polarized electromagnetic fields in a configuration that is invariant in the $z$-direction. An object with a finite support $\mathbb{D}$ is located in vacuum and is characterized by a conductivity $\sigma$, a permittivity $\varepsilon=\varepsilon_{0} \varepsilon_{\mathrm{r}}$, and a permeability $\mu_{0}$. Clearly, the object has no magnetic contrast and the conductivity $\sigma$ and the relative permittivity $\varepsilon_{\mathrm{r}}$ of the object are unknown. It is our objective to retrieve these medium parameters from scattered electric field data measured outside the support of the object. Specifically, for the scattered field at a receiver location with position vector $\boldsymbol{\rho}=\boldsymbol{\rho}^{\text {rec }} \notin \mathbb{D}$, we have the so-called data equation

$$
E_{z}^{\mathrm{sc}}\left(\boldsymbol{\rho}^{\mathrm{rec}}, \omega\right)=\zeta \frac{\mathrm{i} k^{2}}{4} \int_{\boldsymbol{\rho}^{\prime} \in \mathbb{D}} H_{0}^{(1)}\left(k\left|\boldsymbol{\rho}^{\mathrm{rec}}-\boldsymbol{\rho}^{\prime}\right|\right) E_{z}\left(\boldsymbol{\rho}^{\prime}, \omega\right) \mathrm{d} A,
$$


where $\mathrm{i}$ is the imaginary unit, $k=\omega / c_{0}$ is the wavenumber $\left(c_{0}\right.$ is the electromagnetic wave speed in vacuum), $H_{0}^{(1)}$ is the Hankel function of the first-kind and order zero, $\zeta$ is the contrast coefficient given by

$$
\zeta=\varepsilon_{\mathrm{r}}-1+\mathrm{i} \frac{\sigma}{\omega \varepsilon_{0}}
$$

and $E_{z}$ in the integrand is the total electric field strength inside the object. The latter field is unknown, but we do know that it has to satisfy the object equation

$$
E_{z}(\boldsymbol{\rho}, \omega)-\zeta \frac{\mathrm{i} k^{2}}{4} \int_{\boldsymbol{\rho}^{\prime} \in \mathbb{D}} H_{0}^{(1)}\left(k\left|\boldsymbol{\rho}-\boldsymbol{\rho}^{\prime}\right|\right) E_{z}\left(\boldsymbol{\rho}^{\prime}, \omega\right) \mathrm{d} A=E_{z}^{\mathrm{inc}}(\boldsymbol{\rho}, \omega),
$$

with $\boldsymbol{\rho} \in \mathbb{D}$ and where $E_{z}^{\text {inc }}$ is the known incident field. Equation (3) is a Fredholm integral equation of the second kind for the total field $E_{z}$ inside the object if the contrast coefficient is known. Also note that the total field in $\mathbb{D}$ is equal to the incident field and the scattered field at the receiver location vanishes if $\zeta=0$ (no object is present in $\mathbb{D}$ ). From this moment on we exclude this trivial case and consider nonzero contrast coefficients only.

We discretize Eqs. (1) and (3) on a uniform grid and use pulse expansions for the electric field strength (see [4], for example). We obtain the discretized data equation

$$
E_{z}^{\mathrm{sc}}\left(\boldsymbol{\rho}^{\mathrm{rec}}, \omega\right) \approx \zeta \mathbf{r}^{T} \mathbf{u}
$$

and the discretized object equation

$$
(\mathbf{I}-\zeta \mathbf{G}) \mathbf{u}=\mathbf{u}^{\text {inc }} .
$$

In these equations, $\mathbf{r}$ is the receiver vector, $\mathbf{u}$ contains the field approximations for the total field inside the object, and $\mathbf{u}^{\text {inc }}$ contains incident electric field values sampled on the grid. Matrix $\mathbf{G}$ is a matrix representation of the Hankel function (Greens function) and its action on a vector can be computed using the FFT. Finally, we call the right-hand side of Eq. (4) modeled scattered field data and set $v^{\mathrm{sc}}=\zeta \mathbf{r}^{T} \mathbf{u}$.

The way we retrieve the permittivity and conductivity of the object is by inspecting a certain objective function. Specifically, we first assume that minimum and maximum relative permittivity and conductivity values can be found such that $\varepsilon_{\mathrm{r} ; \min } \leq \varepsilon_{\mathrm{r}} \leq \varepsilon_{\mathrm{r} ; \max }$ and $\sigma_{\min } \leq \sigma \leq \sigma_{\max }$, where $\varepsilon_{\mathrm{r} ; \max } \geq \varepsilon_{\mathrm{r} ; \min } \geq 1$ and $\sigma_{\max } \geq \sigma_{\min } \geq 0$. The above intervals define our region of interest in the complex $\zeta$-plane. This region is discretized using a uniform grid, and for each grid value we solve the object equation (5) for the total field $\mathbf{u}$. Having found this field, we substitute $\mathbf{u}$ in the expression for $v^{\text {sc }}$ to obtain the corresponding modeled scattered field at the receiver location. We repeat this procedure for all values on the $\zeta$-grid. A collection of modeled scattered fields is obtained in this way and all these fields are compared with the true measured field using the objective function

$$
F=\frac{\left|E_{z}^{\mathrm{sc}}\left(\boldsymbol{\rho}^{\mathrm{rec}}, \omega\right)-v^{\mathrm{sc}}(\omega)\right|^{2}}{\left|E_{z}^{\mathrm{sc}}\left(\boldsymbol{\rho}^{\mathrm{rec}}, \omega\right)\right|^{2}} .
$$

Other, possibly nondifferentiable objective functions, can be used as well. If the modeled scattered field is such that $F<\epsilon$, where $\epsilon>0$ is a user specified tolerance, then the contrast coefficient corresponding to this field is a solution of our inverse problem.

\section{RESIDUAL MINIMIZATION}

The approach outlined in the previous section is practical if we can efficiently solve the discretized object equation for a large number of different contrast coefficients. This can be achieved by exploiting the shift-invariance property of Krylov subspaces. Loosely speaking, what this amounts to is that if we solve the forward problem for a particular worst-case contrast coefficient, then very little extra work is needed to obtain the total field for all other contrast coefficients in our $\zeta$-domain of interest.

We start by introducing the contrast source $\mathbf{w}=-\zeta \mathbf{u}$ (the minus sign is included for convenience only) and we rewrite the object equation as

$$
\mathbf{A}(\alpha) \mathbf{w}=\mathbf{u}^{\text {inc }} \quad \text { where } \quad \mathbf{A}(\alpha)=\mathbf{G}+\alpha \mathbf{I}
$$

and $\alpha=-1 / \zeta$ (recall that $\zeta \neq 0$ ). Suppose now that we use the Generalized Minimum Residual method (GMRES method, see [3]) to solve this system for a particular value of $\alpha$. After $m$ iterations 


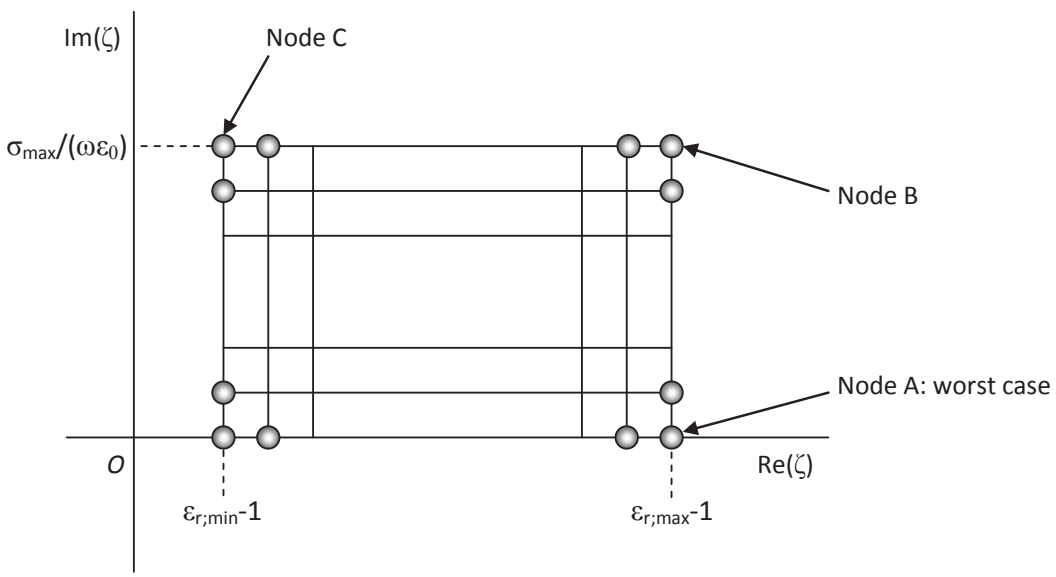

Figure 1: Grid of $\zeta$-values of interest $\left(\sigma_{\min }=0\right)$.

we have the field approximation $\mathbf{w}_{m}$ available and the corresponding residual is given by $\mathbf{r}_{m}=$ $\mathbf{u}^{\text {inc }}-\mathbf{A}(\alpha) \mathbf{w}_{m}$. The question now is: which value of $\alpha$ (or, equivalently, which value of $\zeta$ ) requires the largest number of iterations to reach a prescribed and sufficiently small tolerance level for the normalized 2-norm of the residual? It is reasonable to assume that the contrast coefficient for which $|\zeta|$ is maximum is the worst-case contrast coefficient (node B in Figure 1). This, however, turns out not to be true. Excessive numerical testing shows that the worst-case $\zeta$ is given by $\operatorname{Im}(\zeta)=0$ and $\operatorname{Re}(\zeta)=\varepsilon_{\mathrm{r} ; \max }-1$. In other words, the worst-case configuration consists of a lossless object for which the relative permittivity is maximum (node $\mathrm{A}$ in Figure 1). This is illustrated in Figure 2 where we show the normalized 2-norm of the residual vector for contrast coefficients located at nodes $\mathrm{A}, \mathrm{B}$, and $\mathrm{C}$ in Figure $1\left(\mathbf{r}_{0}=\mathbf{u}^{\text {inc }}\right)$. The object is a square block with a side length equal to the wavelength of the electromagnetic field. Out of the three cases, the contrast coefficient corresponding to node A produces the worst convergence behavior.

Having identified the worst-case contrast coefficient, we write the corresponding $\alpha$ parameter as $\tilde{\alpha}$ and the system of Eq. (7) is written as

$$
[\mathbf{A}(\tilde{\alpha})+\beta \mathbf{I}] \mathbf{w}=\mathbf{u}^{\text {inc }} \quad \text { where } \quad \beta=\alpha-\tilde{\alpha} .
$$

Following [2], we call the above system with $\beta=0$ the seed system, and we call Eq. (8) with a $\beta \neq 0$ an add system. We run the GMRES algorithm only for the worst-case seed system to reach a prescribed tolerance for the normalized 2-norm of the residual vector. The field approximations for the add systems follow by solving small scale least-squares problems. Specifically, after $m$ iterations of the Arnoldi algorithm with the seed system matrix $\mathbf{A}(\tilde{\alpha})$, we have the summarizing equation

$$
\mathbf{A}(\tilde{\alpha}) \mathbf{V}_{m}=\mathbf{V}_{m+1} \tilde{\mathbf{H}}_{m},
$$

where $\tilde{\mathbf{H}}_{m}$ is an $m+1$-by- $m$ upper Hessenberg matrix containing the recurrence coefficients of the Arnoldi algorithm, and the columns of matrix $\mathbf{V}_{m}$ form an orthonormal basis of the Krylov subspace generated by $\mathbf{A}(\tilde{\alpha})$ and the incident field vector $\mathbf{r}_{0}=\mathbf{u}^{\text {inc }}$. Now let $\mathbf{w}_{m}$ belong to this Krylov subspace and take this vector as an approximation to $\mathbf{w}$ in Eq. (8). We write $\mathbf{w}_{m}=\mathbf{V}_{m} \mathbf{c}_{m}$ and the corresponding residual is given by

$$
\mathbf{r}_{m}=\mathbf{u}^{\mathrm{inc}}-[\mathbf{A}(\tilde{\alpha})+\beta \mathbf{I}] \mathbf{w}_{m}=\mathbf{V}_{m+1}\left\{\left\|\mathbf{u}^{\mathrm{inc}}\right\| \mathbf{e}_{1}^{(m+1)}-\left[\tilde{\mathbf{H}}_{m}+\beta\left(\begin{array}{c}
\mathbf{I}_{m} \\
-\mathbf{0}-
\end{array}\right)\right] \mathbf{c}_{m}\right\},
$$

where $\|\cdot\|$ denotes the 2-norm and $\mathbf{e}_{1}^{(m+1)}$ is the first column of the $m$-by- $m$ identity matrix. Since the basis vectors are orthonormal, the 2-norm of the residual is given by

$$
\left\|\mathbf{r}_{m}\right\|=\|\| \mathbf{u}^{\mathrm{inc}}\left\|\mathbf{e}_{1}^{(m+1)}-\left[\tilde{\mathbf{H}}_{m}+\beta\left(\begin{array}{c}
\mathbf{I}_{m} \\
-\mathbf{0}-
\end{array}\right)\right] \mathbf{c}_{m}\right\|
$$

and we observe that for each new value of $\beta$ we need to solve a small scale least-squares problem only. To summarize, GMRES solves the seed and add systems simultaneously, since the Krylov 


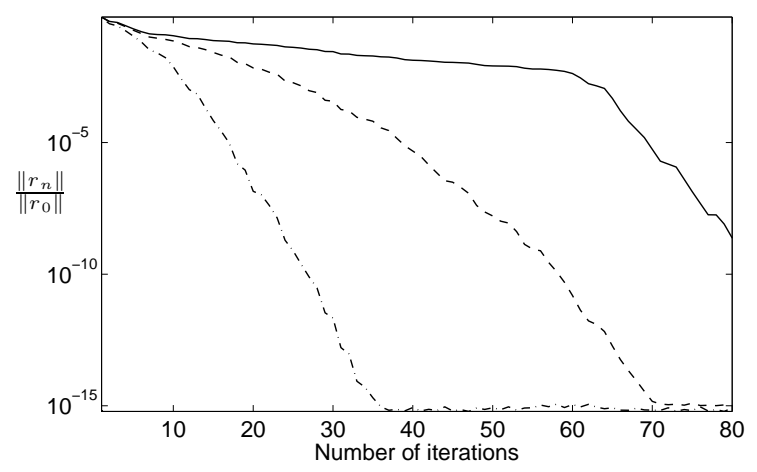

Figure 2: Normalized 2-norm of the residual for contrast coefficients corresponding to node A (solid line), node B (dashed line), and node C (dashed-dotted line).

subspaces generated with $\mathbf{r}_{0}=\mathbf{u}^{\text {inc }}, \mathbf{G}, \mathbf{A}(\tilde{\alpha})$ and $\mathbf{A}(\alpha)=\mathbf{A}(\tilde{\alpha})+\beta \mathbf{I}$ are all the same. More precisely, we have

$$
\mathbb{K}^{m}\left[\mathbf{A}(\tilde{\alpha}), \mathbf{r}_{0}\right]=\mathbb{K}^{m}\left[\mathbf{A}(\alpha), \mathbf{r}_{0}\right]=\mathbb{K}^{m}\left(\mathbf{G}, \mathbf{r}_{0}\right)
$$

and the dimension $m$ is determined by the worst-case contrast coefficient. The object equation for this worst-case coefficient is the seed system and as soon as the 2-norm of the corresponding residual is sufficiently small, we have essentially solved all add systems as well.

The use of Arnoldi's method may become prohibitive for electrically large objects (objects large compared with the wavelength of the electromagnetic field) and problems requiring a large number of Arnoldi iterations, since all Krylov basis vectors need to be stored and the computational load also increases with $m$. Using a restart version of GMRES then comes to mind, but implementing such a procedure is not straightforward, since we may loose the shift-invariance property of the Krylov subspaces. Suppose, for example, that we restart the GMRES method after $p$ iterations. Denoting the residual of the seed and add system by $\mathbf{r}_{p}(\tilde{\alpha})$ and $\mathbf{r}_{p}(\alpha)$, respectively, we have

$$
\mathbb{K}^{m}\left[\mathbf{A}(\tilde{\alpha}), \mathbf{r}_{p}(\tilde{\alpha})\right] \neq \mathbb{K}^{m}\left[\mathbf{A}(\alpha), \mathbf{r}_{p}(\alpha)\right],
$$

in general. In [2] it is proposed to force the residual vectors of the seed and add systems to be collinear, that is, we require that $\mathbf{r}_{p}(\alpha)=\gamma_{p} \mathbf{r}_{p}(\tilde{\alpha})$ for some $\gamma_{p}$. With this condition it is possible to exploit the shift-invariance property of the Krylov subspaces and restarts are possible as well. We have implemented the restart version based on this idea to avoid possible storage problems. For details about the algorithm and a discussion on when the collinearity property exists, see [2].

\section{NUMERICAL RESULTS}

To retrieve the medium parameters of a square block located in vacuum, we illuminate this block by electromagnetic waves with a frequency of $f=42 \mathrm{MHz}$. The side length of the block is equal to $\lambda$, where $\lambda$ is the wavelength in vacuum that corresponds to this frequency. A singlesource/single-receiver unit is symmetrically located above the block. The distance between the block and the source/receiver unit is $\lambda / 2$. Furthermore, the true medium parameters are $\varepsilon_{\mathrm{r}}=4.5$ and $\sigma=5 \mathrm{mS} / \mathrm{m}$ and for the minimum and maximum medium parameters we take $\varepsilon_{\mathrm{r} ; \mathrm{min}}=1$, $\varepsilon_{\mathrm{r} ; \max }=10, \sigma_{\min }=0 \mathrm{mS} / \mathrm{m}$, and $\sigma_{\max }=10 \mathrm{mS} / \mathrm{m}$. Finally, the domain of interest in the complex $\zeta$-plane is discretized using a $40 \times 40$ grid. We therefore need 1599 forward solutions (the scattered and total field for $\zeta=0$ are known). The seed system has a contrast coefficient $\zeta=9$ and there are 1598 add systems to be solved. The resulting objective function on the domain of interest is shown in Figure 3. The true medium parameters are easily recognized, but there are other minima as well. We therefore carry out two additional experiments at two different frequencies, namely, at $f=30 \mathrm{MHz}$ and $f=36 \mathrm{MHz}$. The objective function becomes

$$
F=\sum_{k=1}^{3} m_{k} \frac{\left|E_{z}^{\mathrm{sc}}\left(\boldsymbol{\rho}^{\mathrm{rec}}, \omega_{k}\right)-v^{\mathrm{sc}}\left(\omega_{k}\right)\right|^{2}}{\left|E_{z}^{\mathrm{sc}}\left(\boldsymbol{\rho}^{\mathrm{rec}}, \omega_{k}\right)\right|^{2}} \quad \text { with } \sum_{k=1}^{3} m_{k}=1 .
$$

We take $m_{k}=1 / 3$ for $k=1,2,3$. We evaluated this objective function by computing forward solutions for each frequency separately and for each frequency we used the Arnoldi algorithm with 

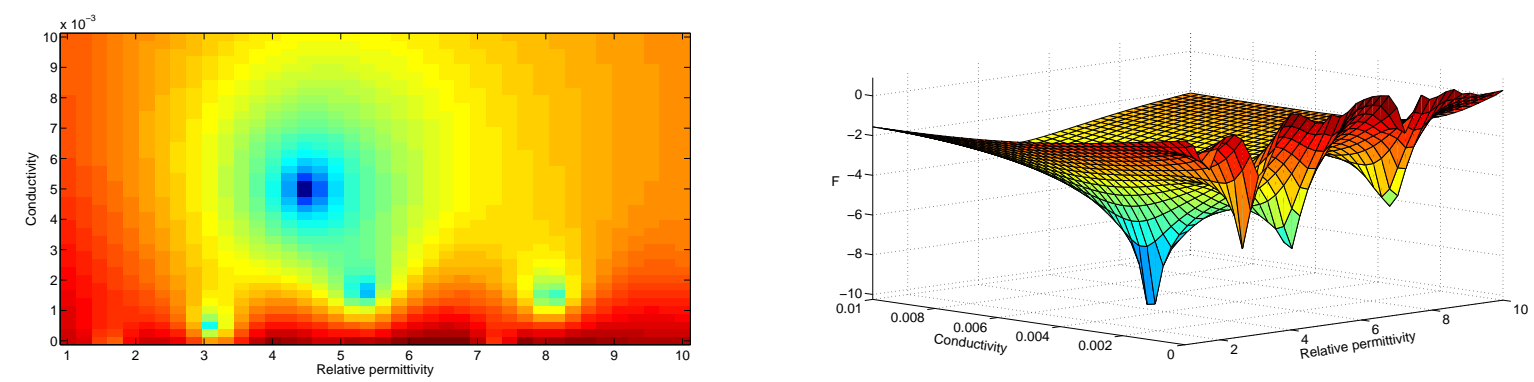

Figure 3: Top view (left) and surface plot (right) of the objective function $F$ on the domain of interest for a single frequency of $f=42 \mathrm{MHz}$.
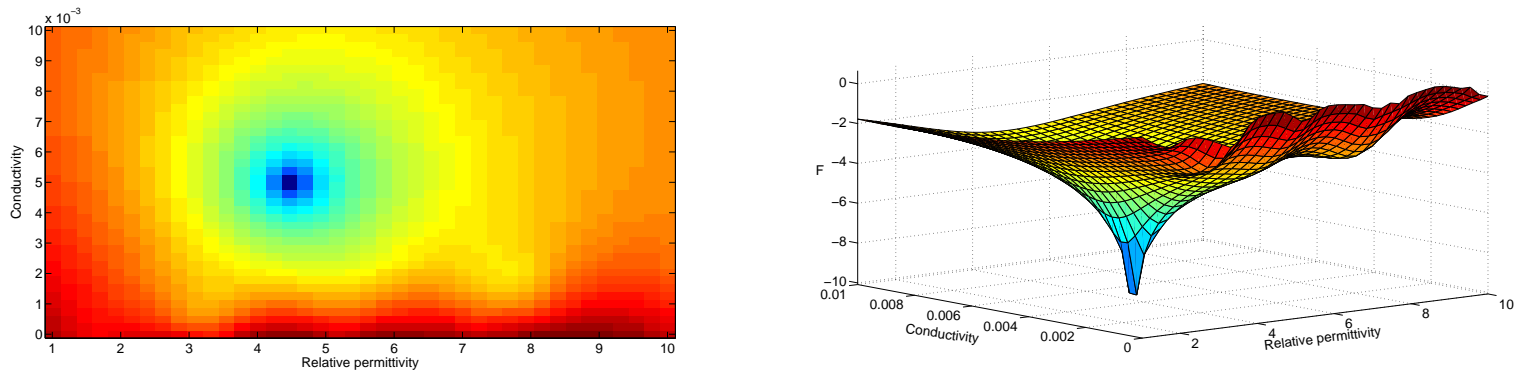

Figure 4: Top view (left) and surface plot (right) of the objective function $F$ on the domain of interest using three frequencies $(f=30, f=36$, and $f=42 \mathrm{MHz})$.

and without restarts to obtain the total fields inside the object. Both approaches produce the same objective function, of course, and this function is shown in Figure 4 . The global minimum corresponding to the true medium parameters remains where it is and a number of false minima have disappeared. We still have local minima around the $\sigma=0$ axis, but the objective function at these minima is much larger than at the global minimum. Moreover, the objective function is smoother than in the single frequency case.

\section{CONCLUSIONS}

We have presented an efficient electromagnetic inversion method for homogeneous objects. The method exploits the shift-invariance property of Krylov subspaces and through residual minimization it very efficiently solves for the total field inside the object. Because of this property, we can solve the inverse problem by simply inspecting a user-defined objective function. Although we have presented our results for a differentiable objective function, other possibly nondifferentiable objective functions may be used as well.

In this paper we have considered homogeneous objects only. For inhomogeneous objects we can still try to match the scattered field due to a homogeneous object to the scattered field generated by an inhomogeneous object. This topic is very important in many different areas most notably in effective medium theory. Presently we are using the technique presented in this paper to investigate to what extent it is possible to find an effective homogeneous scatterer for homogeneous objects with randomly distributed inclusions.

\section{REFERENCES}

1. Budko N. V. and R. F. Remis, "Electromagnetic Inversion Using a Reduced-Order ThreeDimensional Homogeneous Model," Inverse Problems, Vol. 20, No. 6, S17 - S26, 2004.

2. Frommer A. and U. Glässner, "Restarted GMRES for Shifted Linear Systems," SIAM J. Sci. Comput., Vol. 19, No. 1, pp. 15 - 26, 1998.

3. Saad, Y., Iterative Methods for Sparse Linear Systems, Second Edition, SIAM, Philadelphia, 2003.

4. Zwamborn, A. P. M. and P. M. van den Berg, "A Weak Form of the Conjugate Gradient FFT Method for Two-Dimensional TE Scattering Problems," IEEE Trans. Antennas and Propagation, Vol. 39, No. 6, 953 - 960, 1991. 
Appendix B

MATLAB Code 


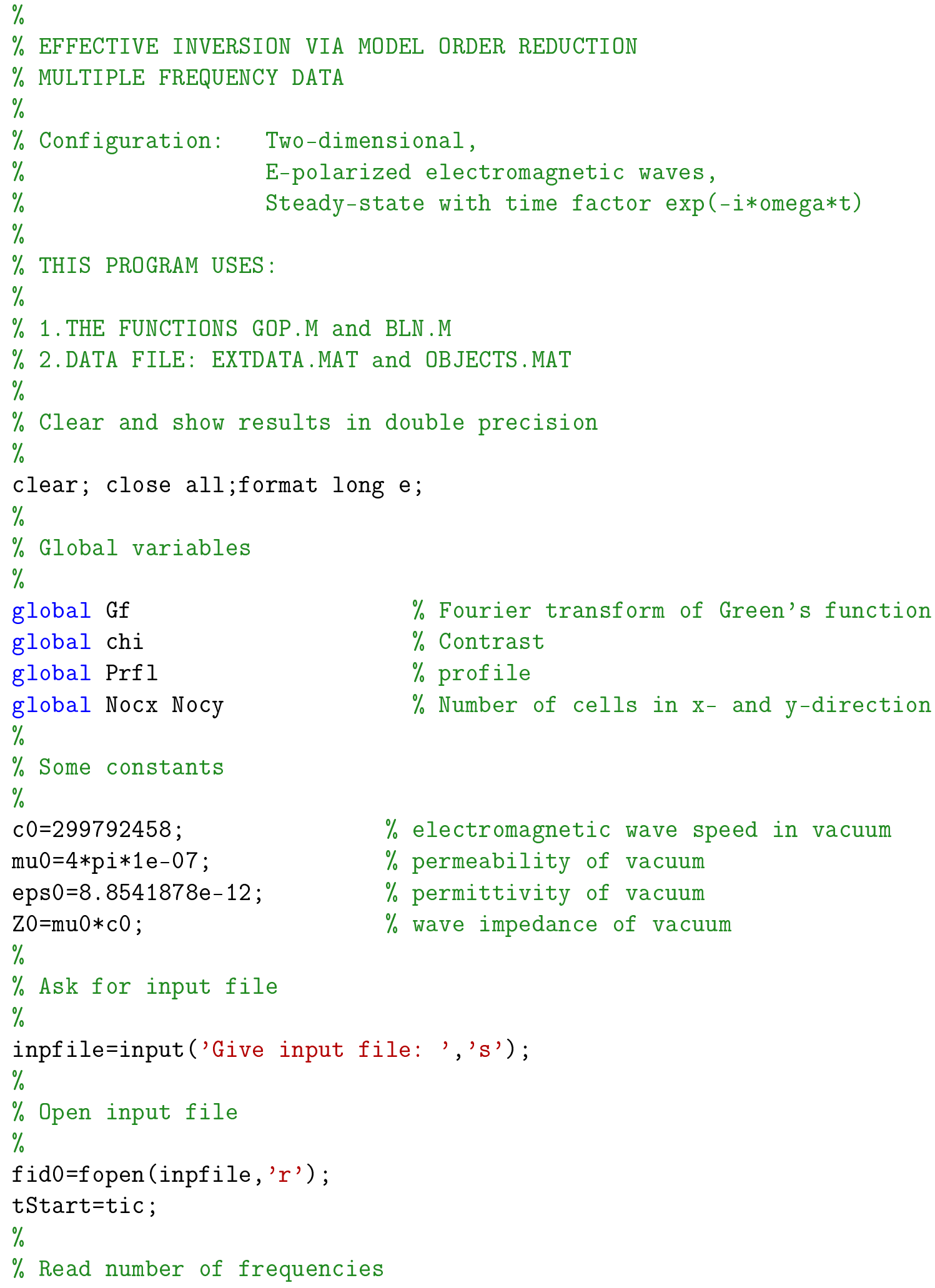




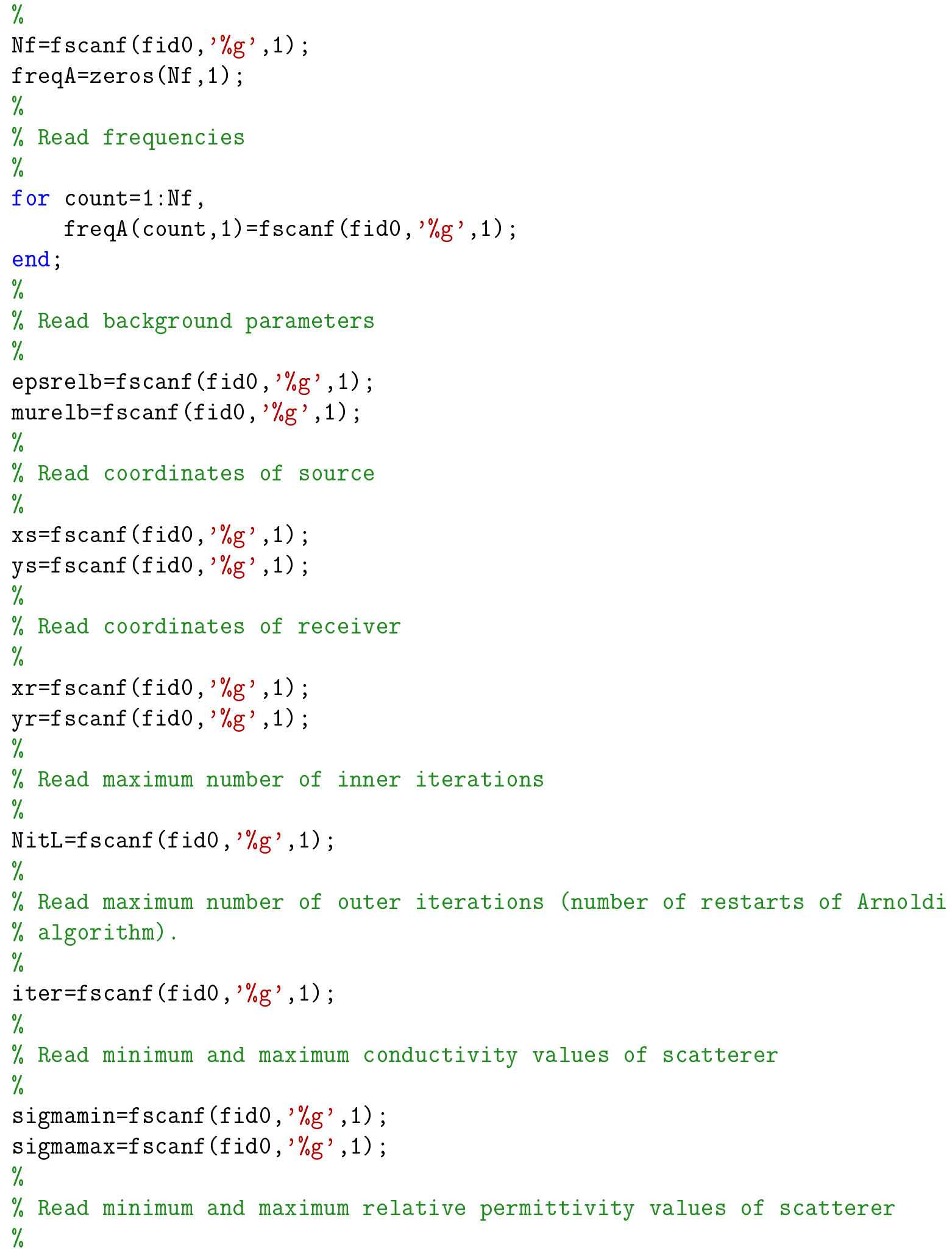




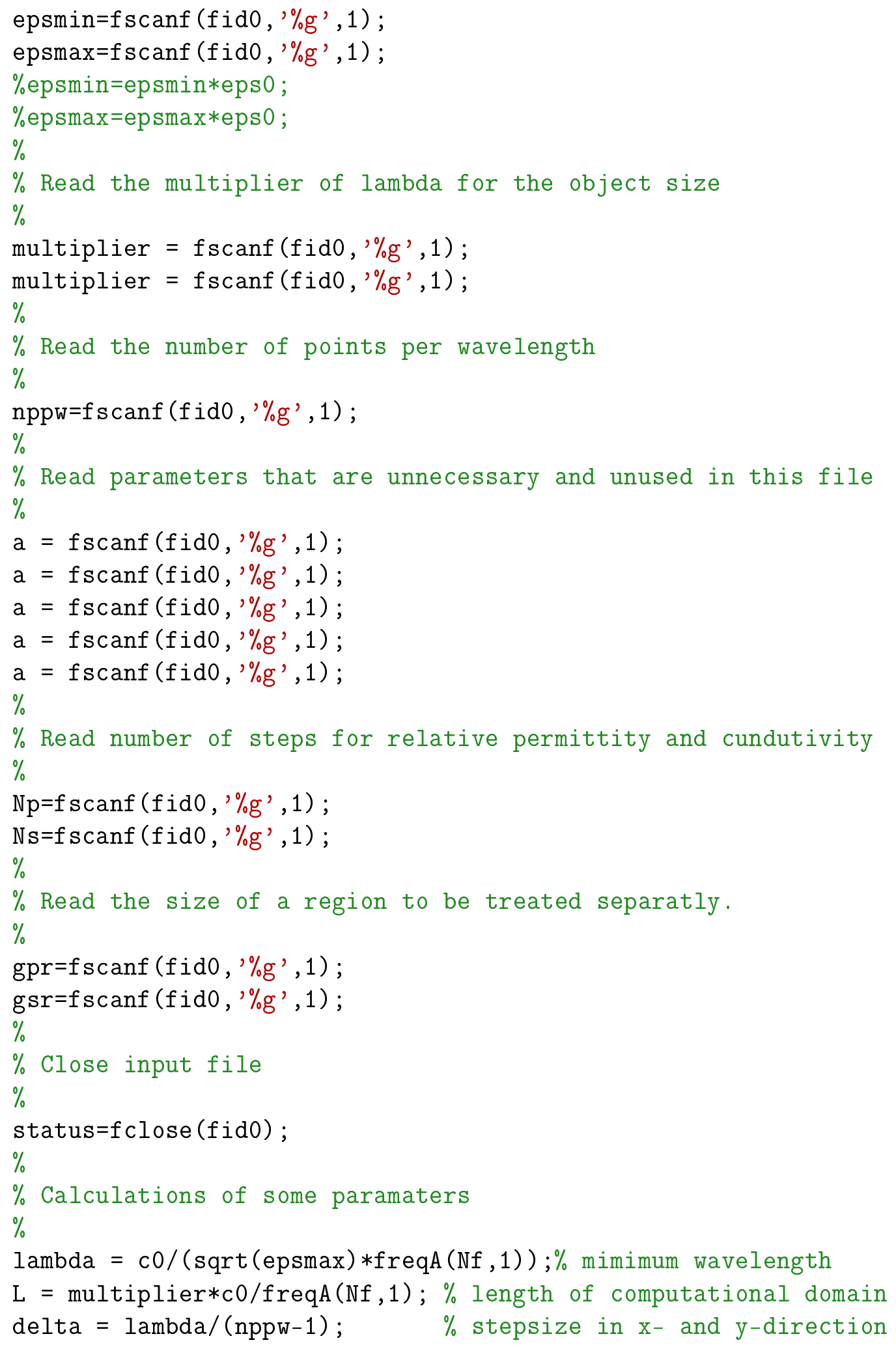


Appendix B - MATLAB Code

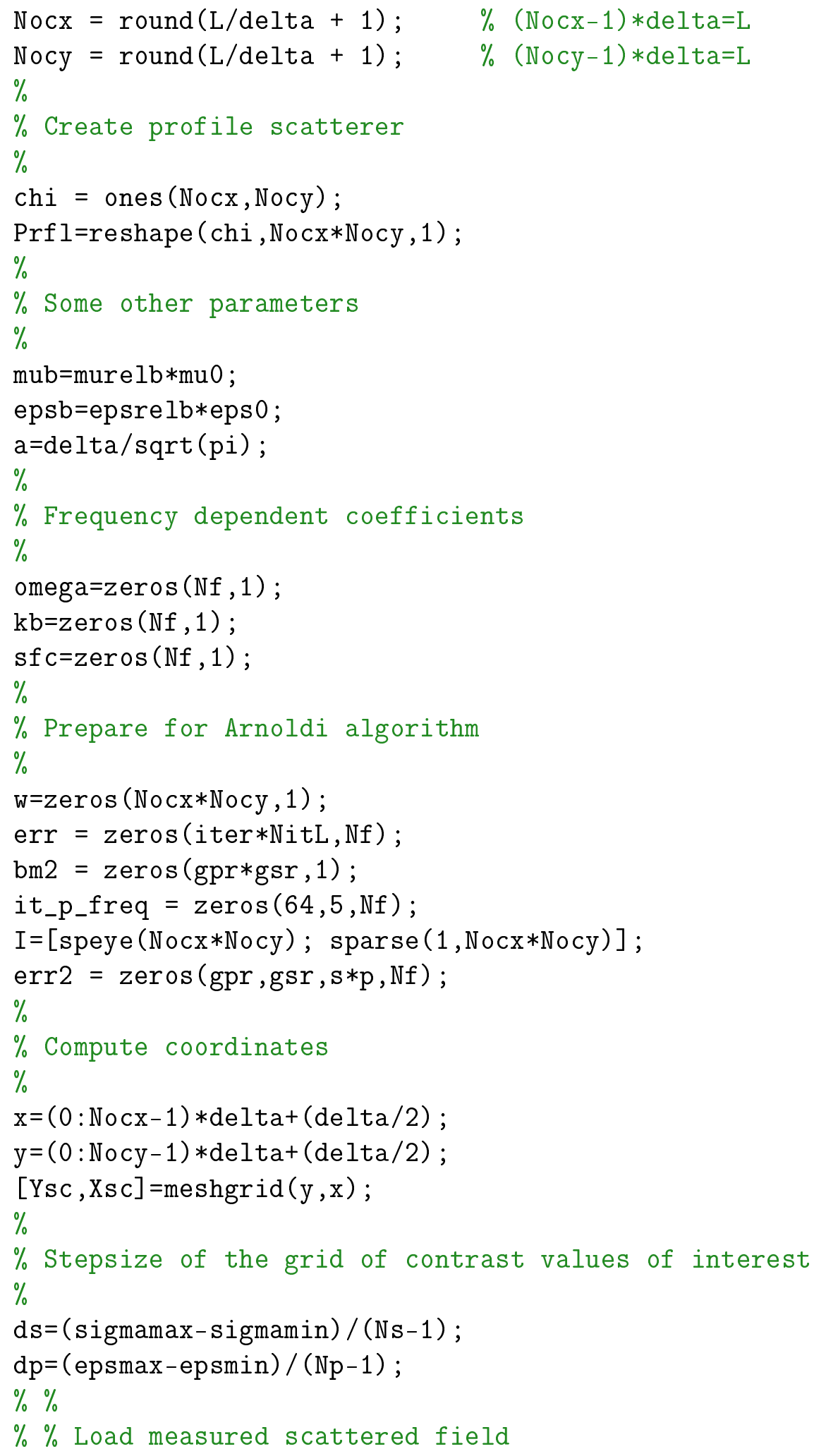


Appendix B - MATLAB Code

$\% \%$

load extdata

$\mathrm{dscr}=\operatorname{zeros}(\mathrm{Np}, \mathrm{Ns})$;

Fobj=zeros (Np, Ns);

dscr_am=zeros (Np, Ns, Nf);

Fobj_am=zeros $(\mathrm{Np}, \mathrm{Ns})$;

$\operatorname{vsc}=\operatorname{zeros}(\mathrm{Nf}, 1)$;

vsC_save=zeros $(\mathrm{Np}, \mathrm{Nf})$;

Gs $=\operatorname{zeros}($ Nocx, Nocy $)$;

\% \% \% \% \% \% \% \% \% \% \% \% \% \% \% \% \% \% \% \% \% \% \% \% \% \% \% \% \% \% \% \% \% \% \% \% \% \% \% \% \% \% \% \% \% \% \% \% \% \% \% \% \% \% \% \% \% \% \% \% \% \% \% \%

$\%$ Partitioning the grid of chi values

$\%$ gpr=region size in permittivity direction

$\%$ gsr=region size in conductivity direction

$\mathrm{s}=\operatorname{ceil}(\mathrm{Ns} / \mathrm{gsr})$;

$\mathrm{p}=\operatorname{ceil}(\mathrm{Np} / \mathrm{gpr}) ;$

$\mathrm{REGG}=\operatorname{zeros}(\mathrm{s}, 2, \mathrm{p})$;

for $\mathrm{k}=1: \mathrm{p}$,

for $i=1: s$,

$\operatorname{REGG}(i,:, k)=[k * g p r i * g s r]$;

end

end

AA1 = squeeze $(\operatorname{REGG}(:, 1,:))$;

$\mathrm{AA1}=$ reshape $(\mathrm{AA} 1, \mathrm{~s} * \mathrm{p}, 1)$;

$\mathrm{AA2}=$ squeeze $(\operatorname{REGG}(:, 2,:)) ;$

$\mathrm{AA2}=\operatorname{reshape}(\mathrm{AA} 2, \mathrm{~s} * \mathrm{p}, 1) ;$

$\mathrm{REGG}=[\mathrm{AA} 1 \mathrm{AA2}]$;

\% \% \% \% \% \% \% \% \% \% \% \% \% \% \% \% \% \% \% \% \% \% \% \% \% \% \% \% \% \% \% \% \% \% \% \% \% \% \% \% \% \% \% \% \% \% \% \% \% \% \% \% \% \% \% \% \% \% \% \% \% \% $\%$

$\%$ Loop over frequencies

$\%$

for count=1:Nf,

freq $=$ freqA (count, 1$)$;

fprintf ('Frequency \#: \%3i \n', count);

$\%$

$\%$ Frequency dependent coefficients

$\%$

omega $($ count, 1$)=2 *$ pi $*$ freq 
Appendix B - MATLAB Code

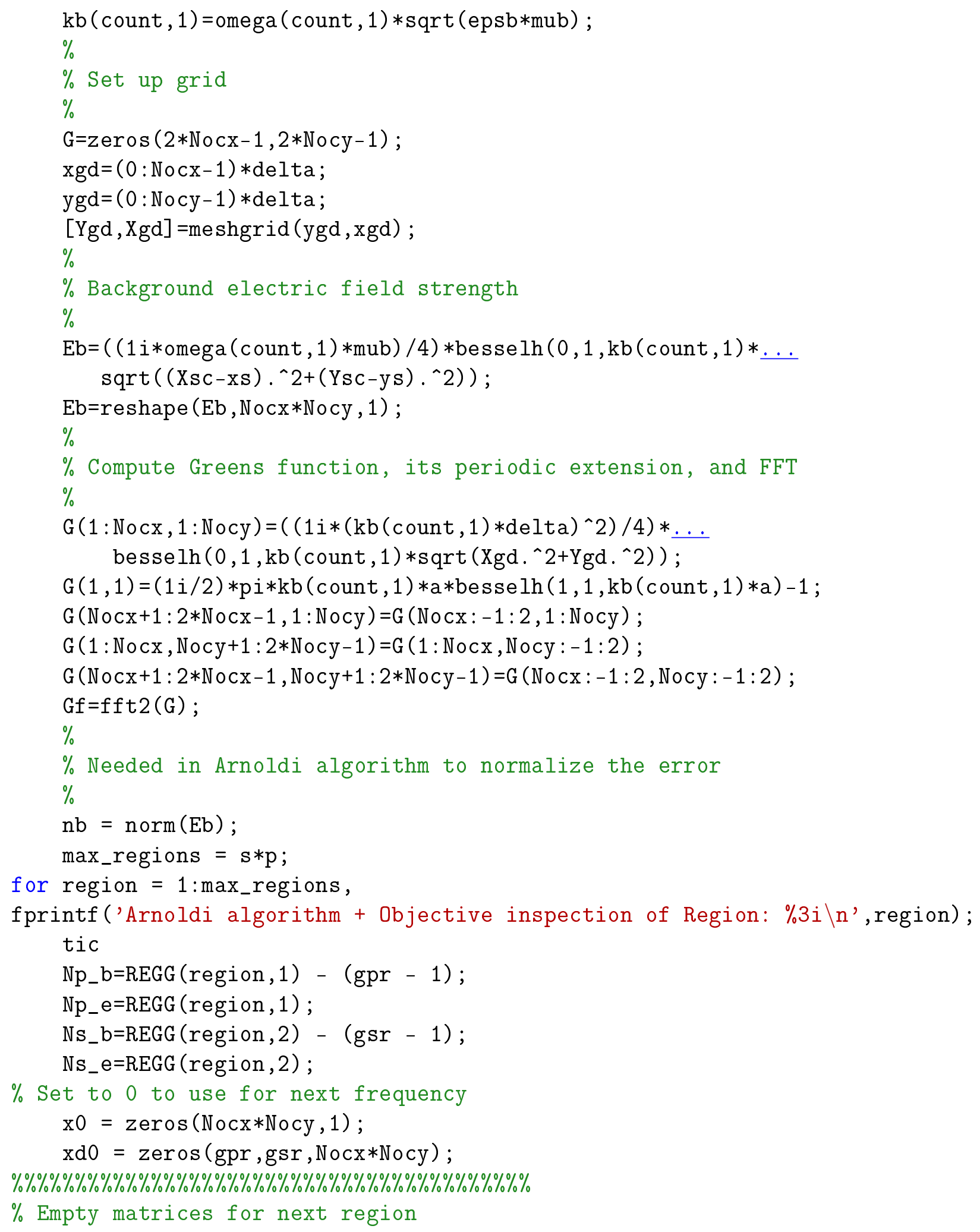


Appendix B - MATLAB Code

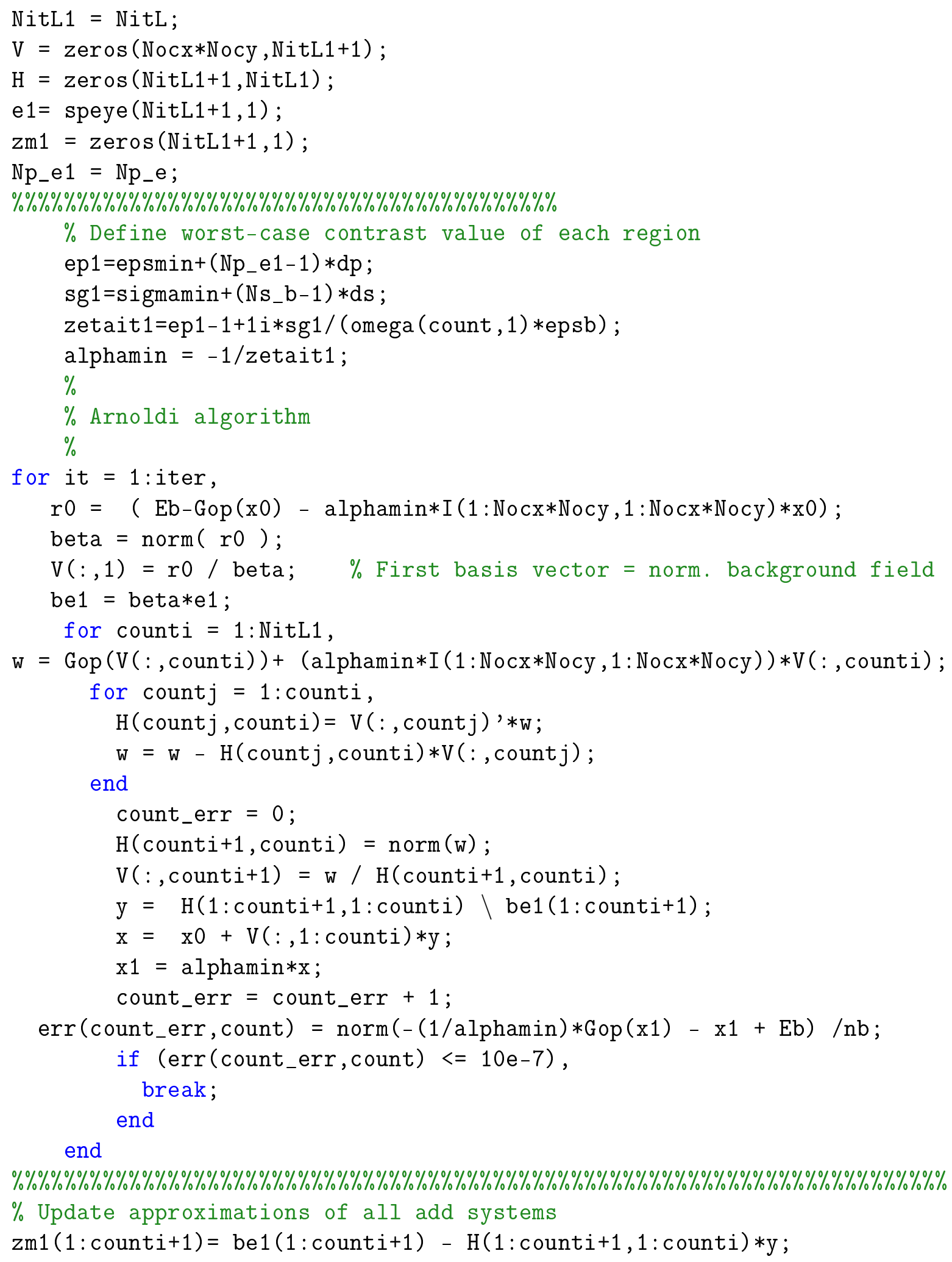


Appendix B - MATLAB Code

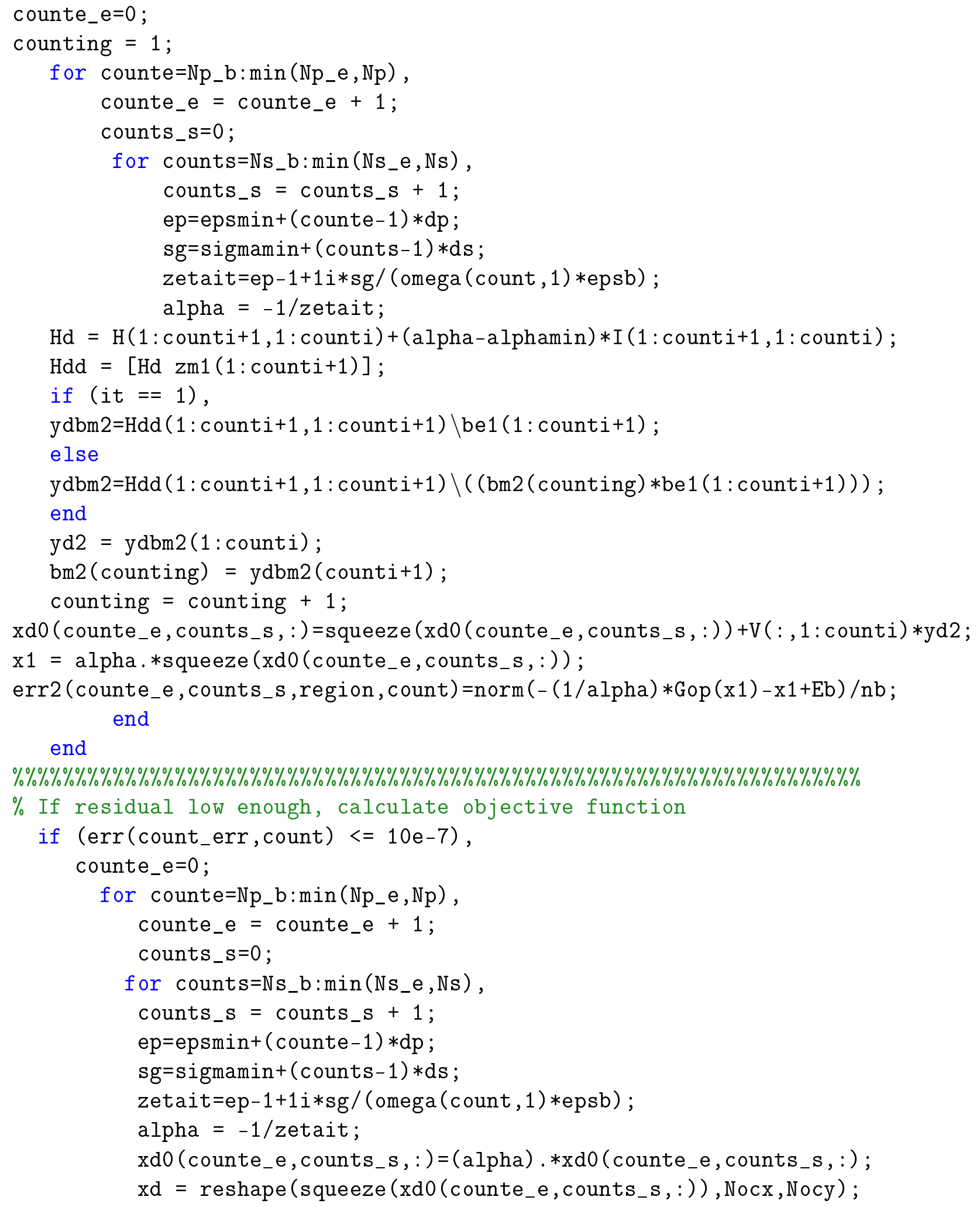


Appendix B - MATLAB Code

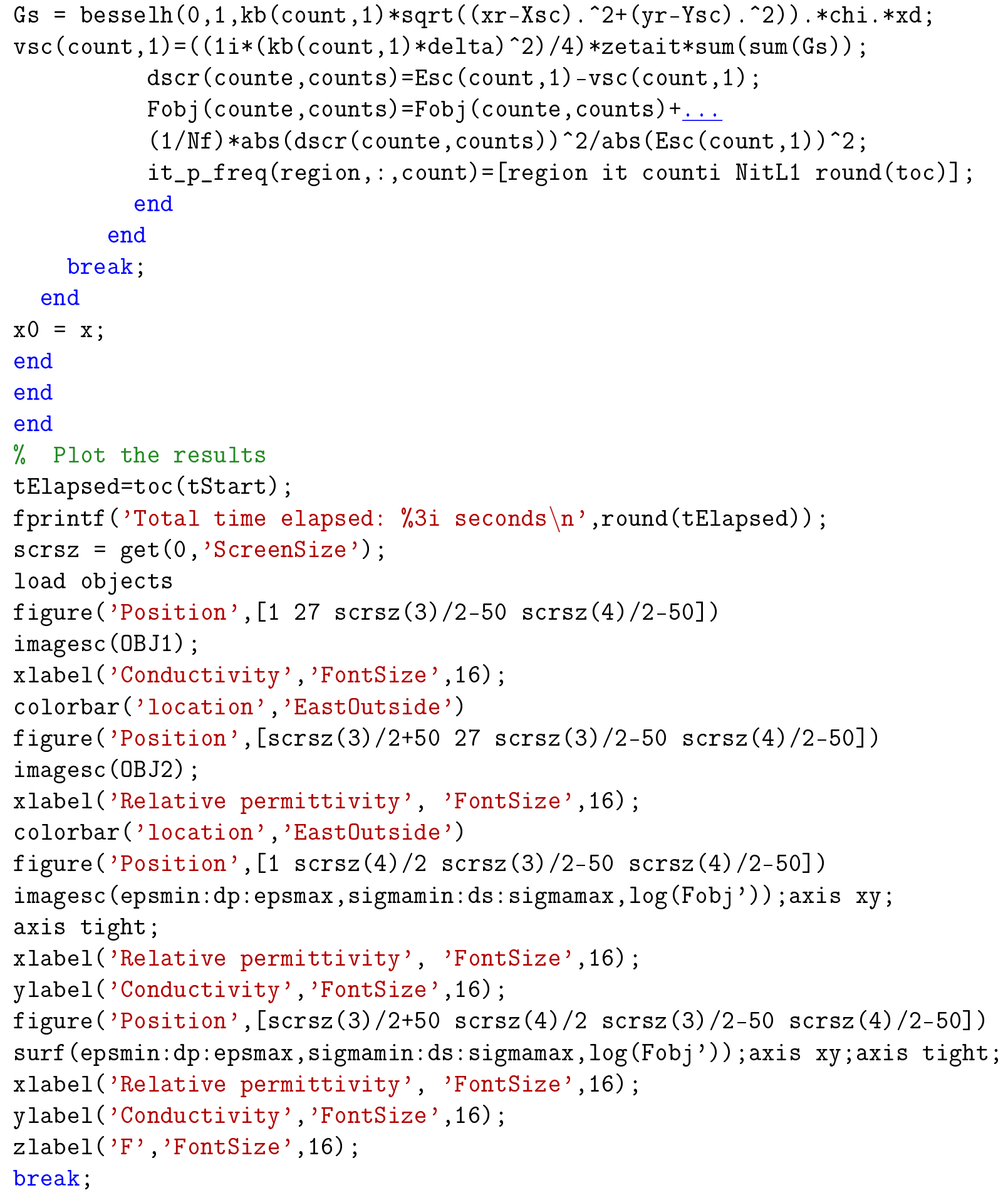




\section{References}

1. Budko, N. V. and R. F. Remis, "Electromagnetic Inversion Using a Reduced-Order Three- Dimensional Homogeneous Model," Inverse Problems, Vol. 20, No. 6, S17 - S26, 2004.

2. Frommer, A. and U. Glässner, "Restarted GMRES for Shifted Linear Systems," SIAM J. Sci. Comput., Vol. 19, No. 1, pp. 15 - 26, 1998.

3. Budko, N.V., "Electromagnetic radiation, scattering and imaging," Lecture notes for the course ET4356, 2004.

4. Verweij, M.D., Berg, P.M. van den. and H. Blok, Electromagnetic Waves, An Introductory Course, DUP Blue Print, 2001.

5. Abramowitz, M. and I. A. Stegun, Handbook of Mathematical Functions, Dover Publications, New York, 1970, p.361.

6. Treffethen, L.N. and D. Bau, Numerical Linear Algebra, SIAM, Philadelphia, 1997.

7. Saad, Y., Iterative Methods for Sparse Linear Systems, Second Edition, SIAM, Philadelphia, 2003.

8. Greenbaum, A., Iterative methods for solving linear systems, SIAM, Philadelphia, 1997 